\title{
Considerations for the Observability of Kinematically Offset Binary AGN
}

\author{
Luke Zoltan Kelley ${ }^{1 \star}$ \\ ${ }^{1}$ Center for Interdisciplinary Exploration and Research in Astrophysics (CIERA), and \\ Department of Physics $\mathcal{E}$ Astronomy, Northwestern University, Evanston, IL 60208
}

22 May 2020

\begin{abstract}
The gravitational waves from Massive black-hole (MBH) binaries are expected to be detected by pulsar timing arrays in the next few years. While they are a promising source for multimessenger observations as binary AGN, few convincing candidates have been identified in electromagnetic surveys. One approach to identifying candidates has been through spectroscopic surveys searching for offsets or time-dependent offsets of broad emission lines (BLs), which may be characteristic of Doppler shifts from binary orbital motion. In this study, we predict the parameter space of MBH binaries that should be kinematically detectable. There is a delicate trade-off between requiring binary separations to be large enough for BL regions to remain attached to one of the AGN, but also small enough such that their orbital velocity is detectable. We find that kinematic signatures are only observable for the lower-mass secondary AGN, for binaries with total-masses above about $10^{8} \mathrm{M}_{\odot}$, and separations between 0.1 and $1 \mathrm{pc}$. We motivate our usage of a kinematic-offset sensitivity of $10^{3} \mathrm{~km} \mathrm{~s}^{-1}$, and a sensitivity to changing offsets of $10^{2} \mathrm{~km} \mathrm{~s}^{-1}$. With these parameters, and an Eddington ratio of 0.1 , we find that $0.5 \%$ of binaries have detectable offsets, and only $0.03 \%$ have detectable velocity changes. Overall, kinematic binary signatures should be expected in fewer than one in $10^{4} \mathrm{AGN}$. Better characterizing the intrinsic variability of BLs is crucial to understanding and vetting MBH binary candidates. This requires multi-epoch spectroscopy of large populations of AGN over a variety of timescales.
\end{abstract}

Key words: quasars: supermassive black holes, quasars: emission lines, gravitational waves, accretion discs

\section{INTRODUCTION}

Binarity and multiplicity are observed at all astrophysical scales, and binaries of massive black holes (MBHs) are believed to be no exception. MBHs are almost ubiquitously observed in the centers of massive galaxies (Soltan 1982; Kormendy \& Richstone 1995; Magorrian et al. 1998) which form through the hierarchical merger of smaller galaxies (Blumenthal et al. 1984; Davis et al. 1985), and continue to merge throughout their lifetimes (Lacey \& Cole 1993; Guo \& White 2008; Lotz et al. 2011; Newman et al. 2012; Rodriguez-Gomez et al. 2015). Two MBHs that are brought together through galaxy merger are only able to form a gravitationally-bound MBH binary (MBHB), and possibly eventually coalesce, through extended dissipative interactions with their local galactic environment (Begelman et al. 1980, i.e., dynamical friction, stellar slingshots, circumbinary-disk torques). Only in a fraction of systems, and over the course of gigayears, do MBHBs reach sufficiently small separations $\left(\lesssim 10^{-2} \mathrm{pc}\right)$ for gravitationalwave $(\mathrm{GW})$ emission to become effective and the system to coa-

\footnotetext{
^ E-mail:1zkelley@northwestern.edu
}

lesce (Rajagopal \& Romani 1995; Sesana et al. 2004; Milosavljević \& Merritt 2003; Merritt \& Milosavljević 2005; Kelley et al. 2017a).

During the final millions of years of inspiral, MBHBs produce GW signals detectable by pulsar timing arrays (Sazhin 1978; Detweiler 1979; Hellings \& Downs 1983; Foster \& Backer 1990) likely within the next few years (Rosado et al. 2015; Taylor et al. 2016; Kelley et al. 2017b; Mingarelli et al. 2017; Kelley et al. 2018). A number of electromagnetic signatures of MBHs in binaries have been suggested when one or both $\mathrm{MBH}$ is accreting and observable as an active galactic nucleus (AGN) or quasar (e.g., Komossa 2006; Popović 2012; Bogdanović 2015; Kelley et al. 2019a; De Rosa et al. 2019). To date, it is still debated whether any 'confirmed' binaries have been observed, but the dual radio cores of 4C37.11 (Rodriguez et al. 2006; Bansal et al. 2017) and the periodic flares of OJ-287 (Sillanpää et al. 1988; Valtonen et al. 2008; Laine et al. 2020; cf. Abraham 2000; Agudo et al. 2012; Britzen et al. 2018) are increasingly convincing. A growing sample of AGN in merging galaxies and dual-AGN, typically at $\gtrsim$ kpc separations (i.e. not gravitationally bound), have been identified. These results suggest that AGN activity increases over the course of galaxy mergers (Bennert et al. 2008; Koss et al. 2012; Comerford \& Greene 
2014; Goulding et al. 2018), consistent with galaxy models and observations (Sanders et al. 1988; Barnes \& Hernquist 1991, 1992).

Even before the underlying nature of AGN was solidified, it was suggested that the emission lines characteristic of these objects could be used to identify them in binaries (Komberg 1968; Gaskell 1983a). As in a stellar spectroscopic-binary, Doppler shifts due to the orbital motion can create a kinematic offset between the AGN line-center and the host-galaxy rest frame. Narrow emission lines (NELs), with velocity widths $\lesssim 10^{3} \mathrm{~km} \mathrm{~s}^{-1}$ and inferred emitting region sizes $\gtrsim 100$ pc (e.g., Gaskell 1983b; Antonucci 1993), are likely only produced at distances beyond where a MBH companion would be bound ${ }^{1}$. Broad emission lines (BLs) have widths of $\sim 10^{4} \mathrm{~km} \mathrm{~s}^{-1}$ and emitting regions $\lesssim \mathrm{pc}$ (Ibid.), and thus can remain bound and co-move with an AGN inside the binary orbit. By comparing the centroids of BLs to host stellar absorption or narrow emission lines, many kinematically-offset binary candidates have been put forward (Dotti et al. 2009; Tsalmantza et al. 2011; Eracleous et al. 2012b; Decarli et al. 2013; Ju et al. 2013; Shen et al. 2013; Liu et al. 2014; Runnoe et al. 2015).

The time variability of BLs has also been used to indicate possible binary motion (Gaskell 1996; Eracleous et al. 2012b; Bon et al. 2012; Ju et al. 2013; Shen et al. 2013; Wang et al. 2017; Liu et al. 2014; Guo et al. 2019) and to exclude or constrain the presence of companions (Halpern \& Filippenko 1988; Eracleous et al. 1997b; Liu et al. 2016; Runnoe et al. 2017; Doan et al. 2020; Lu \& Lin 2019). Another possible avenue of detection is systems with double-peaked BLs, which may be produced by the two components of a binary AGN moving relative to each other (Gaskell 1983a). Some studies have put forward candidates from double-peaked BL sources (Stockton \& Farnham 1991; Eracleous \& Halpern 1994; Boroson \& Lauer 2009; Popović 2012; Decarli et al. 2013; Tsai et al. 2013), while many others suggest these are more likely produced by single AGN (Eracleous et al. 1997a; Eracleous \& Halpern 2003; Strateva et al. 2003; Storchi-Bergmann et al. 2003; Eracleous et al. 2009; Liu et al. 2016; Doan et al. 2020). Keplerian rotation from a disk, for example, is known to produce double-peaks that match observations (e.g., Eracleous et al. 1997a).

Recently, a number of pioneering theoretical studies have carefully modeled the BL characteristics of binary AGN (Bogdanović et al. 2008; Shen \& Loeb 2010a; Nguyen \& Bogdanović 2016; Nguyen et al. 2019b,a). Studies using similar BLRphotoionization models have also specifically targeted the reverberation mapping signatures of binary AGN (Wang et al. 2018; Du et al. 2018; Kovačević et al. 2020), and changes to flux-ratios of lines due to the presence of a binary (Montuori et al. 2011, 2012). Despite these precision modeling studies, a broader view of the accessible binary population and their parameters has only recently been quantified. Ju et al. (2013) and Pflueger et al. (2018) use models of viscous + GW driven binary evolution to calculate the probability of kinematic detectability based on line-of-sight velocities of each binary component. In Pflueger et al. (2018), the authors find a sweet spot of detectability for mass $\operatorname{ratios}^{2} q \equiv M_{2} / M_{1}$ between 0.2 and 0.4 , and binary separations between $10^{3}$ and $10^{4}$ gravitational radii, $r_{g} \equiv G\left(M_{1}+M_{2}\right) / c^{2}$. Detectable systems constitute $\approx 1 \%$ of their overall binary population.

The goal of the current study is to determine what types of

\footnotetext{
1 Although they are still useful for identifying dual-AGN in the same way (e.g. Comerford et al. 2009, 2012; cf. Shen et al. 2011).

2 Note that this is likely due in part to their population priors.
}

MBHB systems are likely to be detectable from kinematic ${ }^{3}$ signatures from optical AGN spectra. We expand on previous work by 1) considering the competing processes that govern the observability of kinematic offsets, 2) determining the limiting plausible sensitivity of surveys to kinematic signatures, and 3) utilizing selfconsistently derived populations of binary AGN. We emphasize the critical importance of observed BLR sizes, which are not included in the analysis of Pflueger et al. (2018). A lower-limit to viable binary separations is established by requiring that the BLRs remain bound to each AGN. This criteria leads to an effective maximum velocity offset, which acts against requirements based on survey sensitivity. This effect is included in the analysis of Ju et al. (2013), but the study does not include a realistic population of systems, and is largely framed in the context of making constraints under the assumption that all AGN are in MBH binaries.

In our study, we find that the resulting, viable parameter space of detectable binaries is very narrow. Kinematic signatures are only detectable in the lower-mass secondary AGN, in systems with relatively extreme mass ratios: $q \lesssim 10^{-2}$, and at separations between 0.1 and $1 \mathrm{pc}\left(r_{g} \approx 10^{3}-10^{4}\right)$. We apply our selection criteria to a population of MBHBs that are derived from cosmological hydrodynamic simulations and evolved using a suite of semi-analytic binary evolution models. While this population suffers from substantial uncertainties, it is a far more comprehensive and self-consistent approach than has been used for kinematic studies in the past. From this population we present plausible binary parameters and detection rates that take into account the intrinsic distribution of sources and survey selection effects. Ultimately, only a very small fraction of all MBHBs are plausibly detectable: one in $\sim 10^{2}$ for velocity offsets, and one in $\sim 10^{4}$ when time-variability is also required.

\section{DETECTABLE PARAMETER SPACE}

\subsection{The BLR Radius and Truncation}

AGN exhibit fairly tight scaling relationships between their luminosity and the characteristic ${ }^{4}$ radius of the BLR (Koratkar \& Gaskell 1991; Kaspi et al. 1996, 2000), generally of the form,

$$
R_{\mathrm{BLR}}=\alpha\left(\frac{L}{L_{0}}\right)^{\beta} .
$$

Relations of this nature are believed to result from the BLR being limited to just within the dusty-torus: determined by the dust sublimation radius, which is proportional to the incident flux (e.g., Barvainis 1987; Netzer \& Laor 1993). Bentz et al. (2013) find best fitting values for the BLâĂŞ $H_{\beta}$ radiusâĂŞluminosity relationship as, $\alpha_{H_{\beta}}=2.8 \times 10^{-2} \mathrm{pc}$ and $\beta_{H_{\beta}}=0.533$, where the luminosity is measured at $5100 \AA$ and normalized to $L_{0}=\lambda L_{\lambda}=10^{44} \mathrm{erg} \mathrm{s}^{-1}$. The radius-luminosity relationship is observed to hold quite well over a broad range of AGN parameters, for example up to eight orders of magnitude in luminosity (e.g., Kaspi et al. 2007). Note that in this section of our analysis, we use best-fitting scaling-relationships

\footnotetext{
3 We prefer 'kinematic' over 'spectroscopic' because non-kinematic, spectroscopic methods of detection exist (e.g. line-ratios, and spectral deficits from disk gaps).

4 Note that the 'BLR radius' is weighted by its emissivity, and in the case of reverberation mapping, also its responsivity to continuum-flux variations. As always, there is also likely bias in the systems with measurements, e.g. towards high Eddington fractions (e.g., Brotherton et al. 2015).
} 

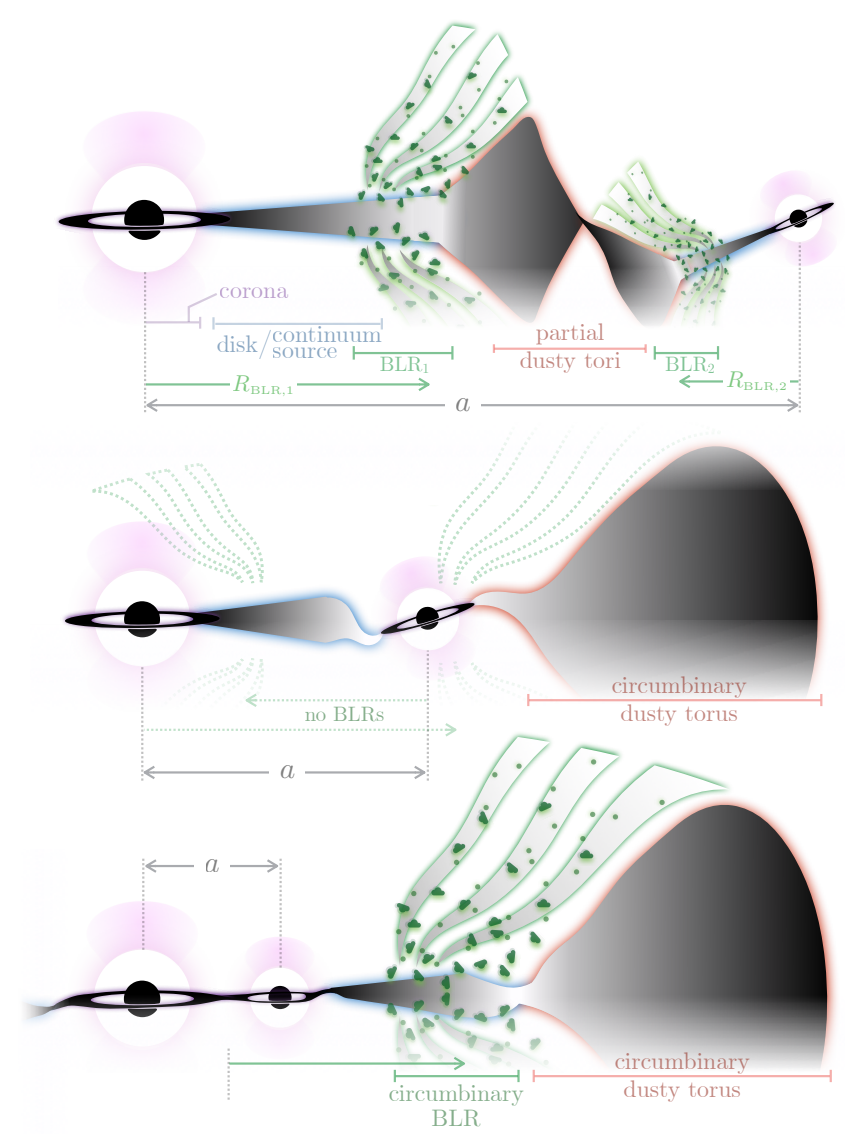

Figure 1. Schematic of Binary AGN and their broad line regions in different regimes. Top: $R_{\mathrm{BLR}}<R_{\mathrm{Hill}}<a$, where BLRs move with each AGN in their orbit. Here, the dusty tori are near the hill radii and are partially disrupted. Middle: $R_{\mathrm{Hill}}<R_{\mathrm{BLR}} \approx a$, where one or both BLRs are disrupted by the binary companion, and the dusty torus is part of the circumbinary disk. Bottom: $R_{\mathrm{BLR}}>R_{\mathrm{Hill}}$, both the torus and BLR are in the circumbinary disk and do not follow the orbital motions of the individual AGN. Only in the top configuration can offset BLs or changing velocities be detected. In either the top or middle panel, the dusty torus may or may not be present. Note that the BLR radii (and emission strengths) are proportional to luminosity, which may not be proportional to each AGN's mass.

without scatter, but the effects of measured scatter are small and discussed later. Using the bolometric corrections from Runnoe et al. (2012),

$$
\lambda_{5100} L_{\lambda, 5100} \approx 2.6 \times 10^{45} \mathrm{erg} \mathrm{s}^{-1}\left(\frac{M_{i}}{10^{8} \mathrm{M}_{\odot}} \frac{f_{\text {Edd }}}{0.1}\right)^{1.1},
$$

combined with Eq. 1 gives,

$$
R_{\mathrm{BLR}} \approx 0.16 \mathrm{pc}\left(\frac{M_{i}}{10^{8} \mathrm{M}_{\odot}} \frac{f_{\mathrm{Edd}}}{0.1}\right)^{0.59} .
$$

The BLR must be within the Hill sphere of its MBH such that the emitting portion is not truncated by the companion (Paczynski 1977; Lin \& Papaloizou 1979). This is shown schematically in Fig. 1. The upper panel shows a wide-separation binary in which the Hill radii of both AGN are outside of the BLR, allowing the BLRs to move with each AGN and thus be observed as kinematically offset. The bottom panel shows a close-binary configuration in which a single, joint BLR forms outside of the binary orbit. This 'circumbinary' BLR will not track the kinematics of the binary AGN components, and will not produce detectable kinematic offsets or variability. In between these regimes, at moderate separations, one or neither BLR may be present, which is depicted in the middle panel.

A fitting formula from Eggleton (1983) gives the effective Hill radius $^{5}$ of each object as,

$$
\frac{R_{\mathrm{Hill}, \mathrm{i}}}{a}=\frac{0.49 q_{i}^{2 / 3}}{0.6 q_{i}^{2 / 3}+\ln \left(1+q_{i}^{1 / 3}\right)} .
$$

Here, $q_{i} \equiv \mu_{i} /\left(1-\mu_{i}\right)$, is the ratio of object $i$ 's mass to that of the other (i.e. the traditional mass-ratio $q=q_{2}=M_{2} / M_{1}$ ), and $\mu_{i} \equiv M_{i} / M=M_{i} /\left(M_{1}+M_{2}\right)$ is each object's mass-fraction. This fit is consistent with both more sophisticated analytic and numerical calculations (see, e.g., Miranda \& Lai 2015). We use Eq. 4 for all of our calculations included in the results, but Artymowicz \& Lubow (1994) give a simpler, and quite accurate ${ }^{6}$ relation that is more convenient for analytic calculations:

$$
\frac{R_{\mathrm{Hill}, 2}}{a}=3^{-2 / 3} \mu_{i}^{1 / 3} \text {. }
$$

Using this approximation, we can set a lower-limit on the semimajor axis to preserve the BLR. Considering that of the secondary $\mathrm{MBH}$ in particular,

$$
\begin{aligned}
a_{\min , 2} & \equiv 3^{2 / 3} R_{\mathrm{BLR}, 2} \mu_{2}^{-1 / 3} \\
& \approx 1.9 \times 10^{-1} \mathrm{pc}\left(\frac{\mu_{2}}{0.1}\right)^{0.25}\left(\frac{M}{10^{8} \mathrm{M}_{\odot}} \frac{f_{\mathrm{Edd}}}{0.1}\right)^{0.59} .
\end{aligned}
$$

We can also express this as a minimum orbital period,

$$
p_{\min } \approx 770 \mathrm{yr}\left(\frac{M}{10^{8} \mathrm{M}_{\odot}} \frac{\mu_{2}}{0.1}\right)^{0.38}\left(\frac{f_{\mathrm{Edd}}}{0.1}\right)^{0.88} .
$$

The minimum orbital period is plotted in Fig. 2, with the additional constraint of a secondary bolometric luminosity above ${ }^{7}$ $10^{43} \mathrm{erg} \mathrm{s}^{-1}$. Even with optimistic parameters for very low-mass MBHBs, orbital periods under $\sim 100 \mathrm{yr}$ are unlikely—and as we discuss later, kinematic offsets from such low masses are likely undetectable.

The BLR radius used here is a characteristic radius. BLR photo-ionization models typically find BLRs that span radii, $R_{\text {outer }} / R_{\text {inner }} \approx R_{\mathrm{BLR}} / R_{\text {inner }} \approx 5-10$ (e.g., Eracleous et al. 1995; Pancoast et al. 2014; Baskin et al. 2014; Baskin \& Laor 2018). These observations suggest that there is not much room to partially truncate the BLR. Dust reverberation mapping studies show that the characteristic radius of the BLR is typically a factor of a few $(\sim 2-$ 5) within that of the dusty torus (e.g., Clavel et al. 1989; Suganuma et al. 2006; Koshida et al. 2014). If emitting BLR-material fills the intervening space, then our usage of the characteristic BLR radius instead of the larger dust-sublimation radius may already be somewhat conservative. Similarly, numerous studies find that the actual truncation radius of a disk could be $25-50 \%$ smaller than the Hill radius (e.g., Pichardo et al. 2005; Martin \& Lubow 2011; Miranda \& Lai 2015). Eating away at the outer portions of the BLR could substantially diminish the line luminosity.

5 This is the Hill radius defined in a volume-averaged sense. How closely the edge of a circumsingle disk approaches the Hill sphere depends on numerous factors, e.g. viscosity and pressure (e.g., Paczynski 1977).

6 The approximation is accurate to better than $95 \%$ for $\mu_{2} \in\left[10^{-4}, 0.5\right]$. For the primary, the expression is accurate to a factor of two, relative to the Eggleton (1983) formula, for $1-\mu_{1} \in\left[10^{-4}, 0.5\right]$.

7 We assume a limiting i-band, $\mathrm{AB}$ magnitude of 22 (e.g., Coffey et al. 2019), which gives a flux of $v_{i} F_{i} \approx 2 \times 10^{-14} \mathrm{erg} / \mathrm{s} / \mathrm{cm}^{2}$. A redshift of $z=0.15$, this corresponds to a luminosity of $\approx 10^{43} \mathrm{erg} \mathrm{s}^{-1}$ using the Runnoe et al. (2012) $5100 \AA ̊$ bolometric correction $(\approx 9.5$ ). 

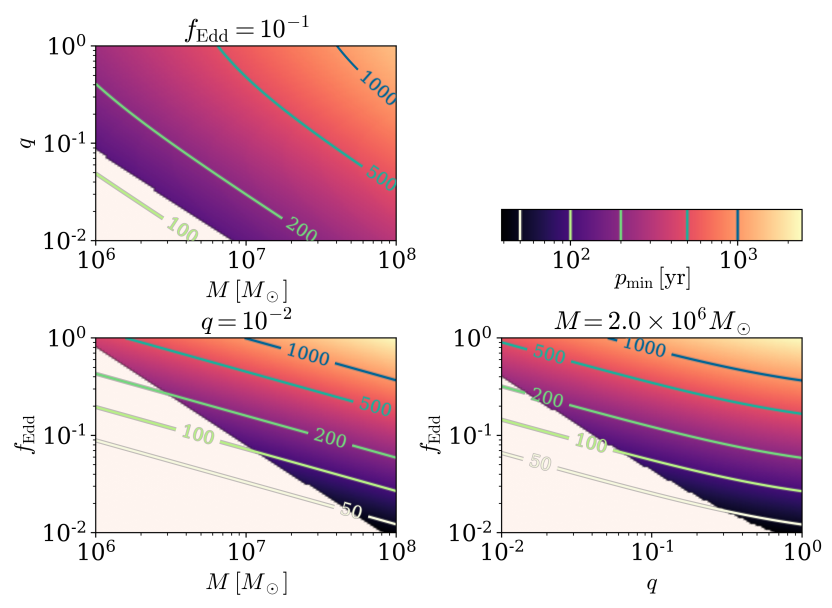

Figure 2. Minimum binary orbital period such that the BLR characteristic radius remains within the Hill sphere of the secondary $\mathrm{MBH}$. The colormap and contours correspond to orbital periods in years. The pale regions in the lower-left of each panel are excluded based on a luminosity threshold of $10^{43} \mathrm{erg} \mathrm{s}^{-1}$ (iband $M_{\mathrm{AB}} \approx 22$ ). The fiducial parameters of $f_{\mathrm{Edd}}=10^{-1}$, $q=10^{-2}$, and $M=2 \times 10^{6} \mathrm{M}_{\odot}$ are chosen to find the smallest orbital periods; such low masses, however, produce very small kinematic offsets that are effectively undetectable.

\subsection{Observable Offsets}

A maximum binary separation (minimum velocity-offset) is determined by requiring that the binary components are bound. This value is determined by the ambient mass density in the galaxy core, which is typically formulated in terms of the nuclear stellar velocity dispersion, $\sigma_{\star}-$ also a minimum orbital-velocity. Keeping in mind that the velocity-dispersion measured by a spectroscopic fiber is only a proxy for the dynamically relevant nuclear velocity dispersion $^{8}$, we calculate velocity-dispersion from the $M_{\mathrm{BH}}-\sigma_{\star}$ of McConnell \& Ma (2013),

$$
\sigma_{\star}=180 \mathrm{~km} \mathrm{~s}^{-1}\left(\frac{M}{10^{8} \mathrm{M}_{\odot}}\right)^{0.177} .
$$

This gives a maximum binary separation of,

$$
a<\frac{G M}{\sigma_{\star}^{2}} \approx 14 \mathrm{pc}\left(\frac{M}{10^{8} \mathrm{M}_{\odot}}\right)^{0.65} .
$$

Again, we note that while scatter in scaling relationships is neglected here, we find that it has little effect our results. Comparing Eq. 9 with Eq. 6 shows that roughly two dex in separation are viable for kinematic MBHB detection. Lowering the Eddington ratio can increase this parameter space to include smaller-separation MBHBs, but this comes at the cost of fainter AGN.

The maximum detectable orbital separation is based on the minimum discernible kinematic velocity-offset. For now, we consider the optimal case of viewing the system within the orbital plane, and at the phase of peak velocity offset. Later, we account for varying inclination and orbital phase. If we parametrize this minimum detectable offset as $v_{\text {sens }}$, then,

$$
a \lesssim 0.35 \mathrm{pc}\left(\frac{M}{10^{8} \mathrm{M}_{\odot}}\right)\left(\frac{v_{\text {sens }}}{10^{3} \mathrm{~km} \mathrm{~s}^{-1}}\right)^{-2}\left(\frac{1-\mu_{2}}{0.9}\right)^{2} .
$$

8 i.e. the fiber may cover a larger (or smaller) solid angle of the galaxy than is relevant, and includes projection effects.
Prospects for detecting kinematic offsets are very sensitive to the particular value of $v_{\text {sens. }}$. A value of $v_{\text {sens }}=10^{3} \mathrm{~km} \mathrm{~s}^{-1}$ is a typical selection criterion employed in the literature (e.g. Eracleous et al. 2012b; cf. Liu et al. 2014), which we explore in \$2.5. Using these fiducial parameters, the maximum separation (Eq. 10) is only slightly larger than the minimum separation (Eq. 6). Comparing the two relations suggests a minimum total mass for a detectable binary,

$$
\begin{aligned}
M_{\min } \approx 2.3 \times 10^{7} \mathrm{M}_{\odot} & \left(\frac{v_{\text {sens }}}{10^{3} \mathrm{~km} \mathrm{~s}^{-1}}\right)^{4.8}\left(\frac{f_{\mathrm{Edd}}}{0.1}\right)^{1.4} \\
& \left(\frac{1-\mu_{2}}{0.9}\right)^{-4.8}\left(\frac{\mu_{2}}{0.1}\right)^{0.61} .
\end{aligned}
$$

This expression for the minimum mass scales with almost the 5th power of the velocity-offset sensitivity, an already uncertain parameter, making the usefulness of this relation somewhat shaky. Note that the strong scaling of the first mass-fraction term makes the minimum mass increase extremely rapidly as the binary approaches equal-mass $\left(\mu_{2} \rightarrow 0.5\right)$.

The accessible parameter space for detecting kinematic offsets is shown in Fig. 3. Each subfigure shows separation (top) and period (bottom) vs. total mass (left) and mass ratio (right). The shaded regions show where the primary (red) and secondary (blue) kinematic offsets are detectable based on 1) the BLR remaining within the Hill spheres, 2) the binary being bound, and 3 ) the orbital velocity being larger than $v_{\text {sens }}$. The top quartet assume a sensitivity to velocity offsets of $v_{\text {sens }}=10^{2} \mathrm{~km} \mathrm{~s}^{-1}$ (e.g., Liu et al. 2014). The bottom set assume a velocity offset sensitivity of $v_{\text {sens }}=10^{3} \mathrm{~km} \mathrm{~s}^{-1}$ (e.g., Eracleous et al. 2012b). Detectable separations are mostly restricted to $\sim\left[10^{-1}, 10^{+1}\right] \mathrm{pc}$, and orbital periods between $\sim\left[10^{3}, 10^{5}\right] \mathrm{yr}$. The detectable parameter space is quite insensitive to mass ratio, though this assumes both a fixed accretion rate, and ignores that the secondary AGN may be outshone by the primary (see $\S 4$ ).

There is a subtlety in how the detectable parameter space changes with varying offset sensitivities that is worth emphasizing. At fixed masses, when $v_{\text {sens }}$ decreases (improves), the minimum detectable separation $\left(a_{\min }\right)$ is unchanged (Eq. 6), and the maximum detectable separation is increased (Eq. 10). At the same time, however, the minimum detectable mass decreases rapidly (Eq. 11), and thus $a_{\min }$ over all masses also decreases. This can be seen in the upper-left sub-panels of Fig. 3, where $a_{\text {min }} \sim 10^{-1} \mathrm{pc}$ for $v_{\text {sens }}=10^{3} \mathrm{~km} \mathrm{~s}^{-1}$, but decreases to $a_{\min } \sim 10^{-2} \mathrm{pc}$ for $v_{\text {sens }}=10^{2} \mathrm{~km} \mathrm{~s}^{-1}$.

Further out in an accretion disk, the velocity gradient decreases until eventually differential rotation is unable to prevent gravitational fragmentation. Numerous other possible instabilities may also limit the disk extent, and estimations of disk bounds can be highly model dependent. The presence of a binary adds significant additional complications. One of the most typically used stability criteria is based on the shear from differential rotation balancing self-gravity - called the Toomre criterion. We illustrate the possibly-unstable regions as shaded grey in Fig. 3, based on the calculation from Haiman et al. (2009), assuming that the singleAGN Toomre stability criterion is roughly valid for a circumbinary disk. In their formalism, the disk is decomposed into three, radiallystratified regions distinguished by their dominant pressure source (radiation or thermal) and opacity (Thompson or free-free). This leads to the two distinct regions of instability in Fig. 3-triangular when plotted against total-mass, and bands when plotted against mass ratio. 

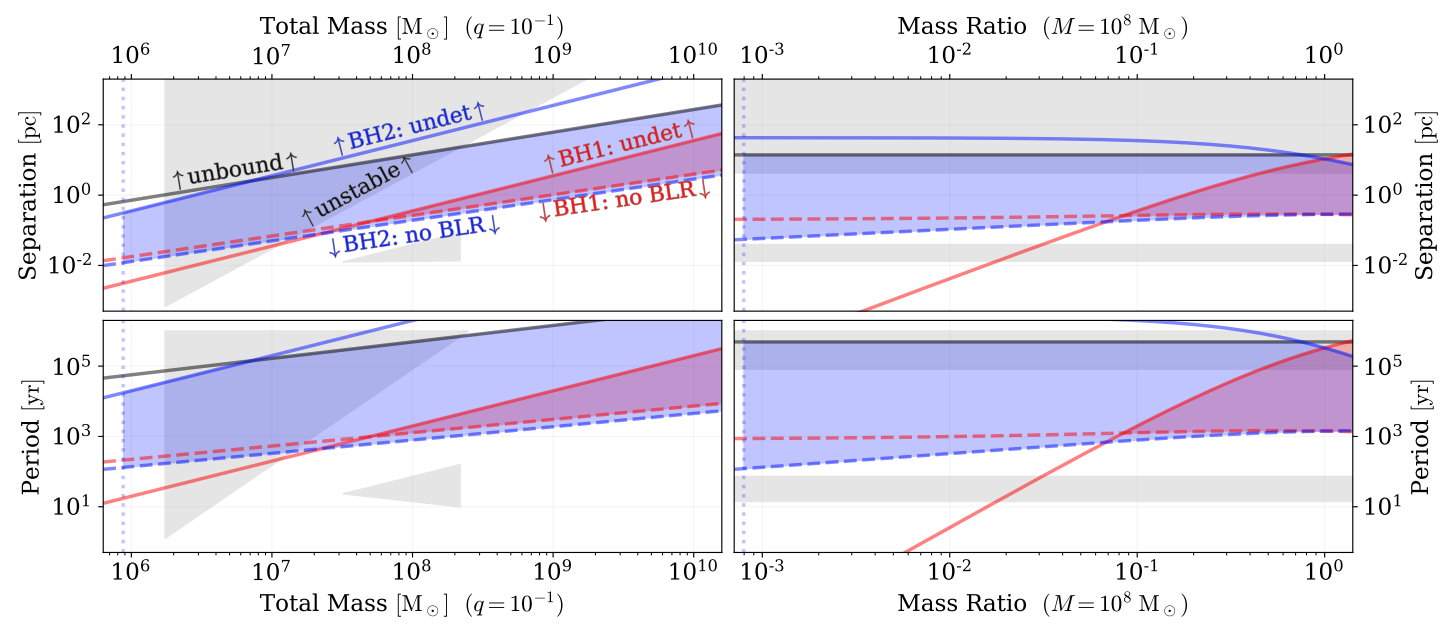

(a) Optimistic velocity-offset sensitivity $v_{\text {sens }}=10^{2} \mathrm{~km} \mathrm{~s}^{-1}$

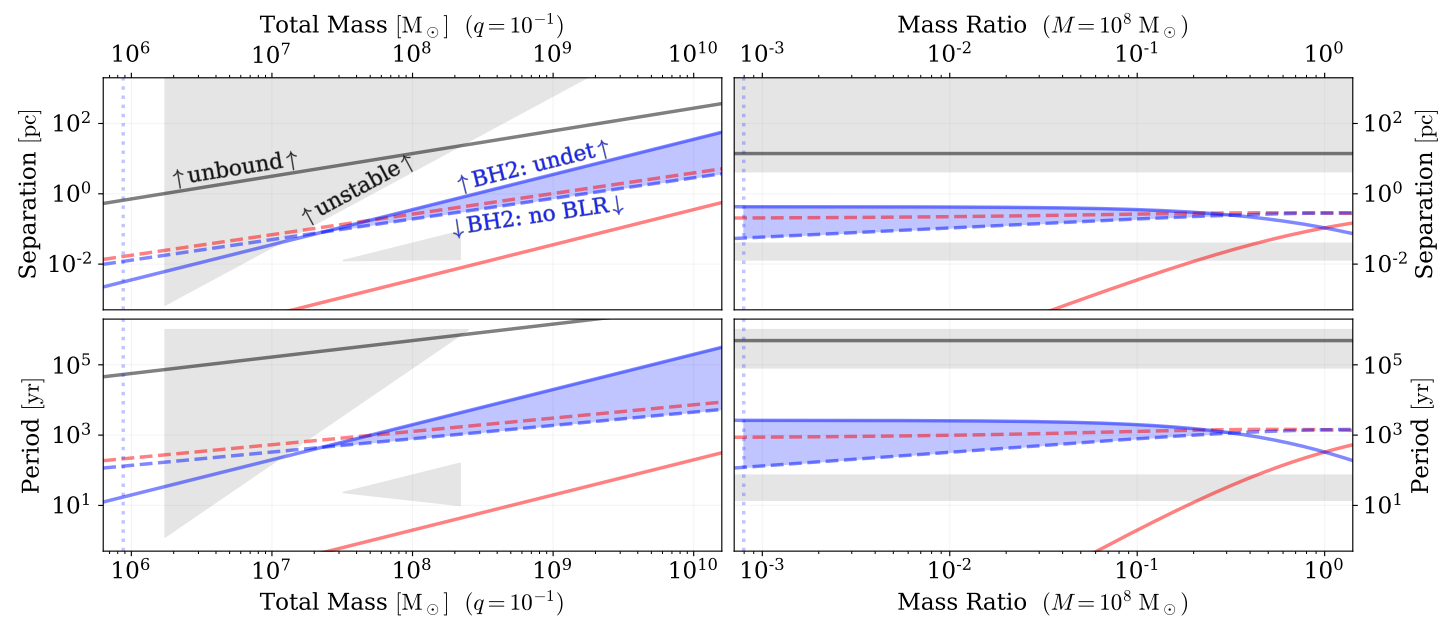

(b) Fiducial velocity-offset sensitivity $v_{\text {sens }}=10^{3} \mathrm{~km} \mathrm{~s}^{-1}$

Figure 3. Binary parameter space accessible by the detection of kinematic BL offsets of the primary (red) and secondary (blue) AGN. In each quartet of panels, orbital separation (top) and period (bottom) are plotted against total mass (left; $q=0.1$ fixed) and mass ratio (right; $M=10^{8} \mathrm{M}_{\odot}$ fixed). We assume an Eddington factor $f_{\text {Edd }}=0.1$ (with a radiative efficiency of 100\%) and a limiting luminosity of $10^{43} \mathrm{erg} \mathrm{s}^{-1}$. Regions are excluded based on the criteria derived in §2: when the BLR is outside of each component's Hill sphere ('no BLR'; dashed lines), the orbital velocity offset is undetectably small ('undet'; solid lines), the binary is 'unbound' (black line), or systems are too faint (vertical, dotted lines). The top set assume a sensitivity to velocity offsets of $v_{\text {sens }}=10^{2} \mathrm{~km} \mathrm{~s}^{-1}$ (e.g., Liu et al. 2014), while the bottom set take $v_{\text {sens }}=10^{3} \mathrm{~km} \mathrm{~s}^{-1}$ (e.g., Eracleous et al. 2012b). The grey shaded regions are Toomre unstable based on the single $A G N$ criteria presented in Haiman et al. (2009); how accurately this applies to binaries is unclear.

It is not obvious that Toomre stability near the binary orbit must be required to feed each AGN's circumsingle disk. However, because we are interested in relatively high-accretion rates $f_{\text {Edd }} \gtrsim 10^{-2}$, it seems reasonable to require a stable circumbinary disk. Requiring Toomre stability at the binary separation is almost always redundant with the sensitivity cut in Eq. 10, when using the fiducial $v_{\text {sens }}=10^{3} \mathrm{~km} \mathrm{~s}^{-1}$ (top quartet of Fig. 3). This is broadly true over a wide range of total-mass and Eddington-ratios. When $v_{\text {sens }}$ is lowered, then smaller binary separations are detectable (as discussed above), but Toomre stability may become an important limitation (bottom quartet of Fig. 3). Overall, only systems with total mass $M \gtrsim 10^{8} \mathrm{M}_{\odot}$ and separations $a \gtrsim 10^{-1} \mathrm{pc}$ are detectable if $v_{\text {sens }} \sim 10^{3} \mathrm{~km} \mathrm{~s}^{-1}$, or if the single-AGN Toomre criterion restricts the parameter space.

\subsection{Observable Changes in Velocity Offsets}

Consider two spectra taken at times separated by $T_{\text {obs }} \ll \tau_{\text {orb }}$, as will generally be the case; and assume that the observer is oriented within the plane of the MBHB's circular orbit. We can estimate the time-change of velocity as $\Delta v \approx(d v / d t) T_{\text {obs }}$, where the acceleration $d v / d t$ must be considered at a particular orbital phase. The maximum change in velocity occurs when both objects are aligned (phase zero for a sinusoidal velocity),

$$
\begin{aligned}
& \Delta v_{i, \max }=T_{\mathrm{obs}}\left(1-\mu_{i}\right)^{2} \frac{G M}{a^{2}} \\
& \Delta v_{2, \max } \approx 180 \mathrm{~km} \mathrm{~s}^{-1}\left(\frac{M}{10^{8} \mathrm{M}_{\odot}}\right)\left(\frac{T_{\mathrm{obs}}}{5 \mathrm{yr}}\right)\left(\frac{a}{10^{-1} \mathrm{pc}}\right)^{-2}\left(\frac{1-\mu_{2}}{0.9}\right)^{2} .
\end{aligned}
$$

Detections will be strongly biased against zero phase, however, because it corresponds to zero velocity offset of the projected orbital motion (Eracleous et al. 2012a). When the velocity offset is max- 
imal (phase $\pm \pi / 2$ ), the observed change in velocity is at its minimum:

$$
\begin{aligned}
& \Delta v_{i, \text { min }}=T_{\mathrm{obs}}^{2}\left(1-\mu_{i}\right)^{3} \frac{(G M)^{3 / 2}}{a^{7 / 2}} \\
& \Delta v_{2, \text { min }} \approx 17 \mathrm{~km} \mathrm{~s}^{-1}\left(\frac{M}{10^{8} \mathrm{M}_{\odot}}\right)^{3 / 2}\left(\frac{T_{\mathrm{obs}}}{5 \mathrm{yr}}\right)^{2}\left(\frac{a}{10^{-1} \mathrm{pc}}\right)^{-7 / 2}\left(\frac{1-\mu_{2}}{0.9}\right)^{3} .
\end{aligned}
$$

While selecting for measurably offset BL centroids, the $\Delta v_{2, \min }$ is a more characteristic value. Identifying systems with both a substantial velocity offset and velocity shift is significantly more difficult than either alone.

Consider a survey in which we search solely for BLRs with changing velocities, regardless of whether the BLR centroid is offset or not. Such a sample would be biased towards the regime in which Eq. 12 is applicable. The strong separation dependence initially seems encouraging because even for a modest sensitivity to velocity changes, small separations would still seem to be highly detectable. Here, however, the separations at which the BLR remains within the Hill spheres of AGN is of critical importance. By assuming some sensitivity to velocity-changes, $\Delta v_{\text {sens }}$, we can compare the minimum allowed separation (s.t. $a>R_{\mathrm{Hill}}>R_{\mathrm{BLR}}$; Eq. 6) to the maximum separation that is detectable (s.t. $\Delta v_{2, \min }>\Delta v_{\text {sens }}$; Eq. 13):

$$
\begin{aligned}
\frac{a_{\mathrm{max}, \Delta v}}{a_{\min }} \approx 0.70 & \left.\frac{T_{\mathrm{obs}}}{5 \mathrm{yr}}\right)^{1 / 2}\left(\frac{\Delta v_{\mathrm{sens}}}{100 \mathrm{~km} \mathrm{~s}^{-1}}\right)^{-1 / 2}\left(\frac{M}{10^{8} \mathrm{M}_{\odot}}\right)^{-0.086} \\
& \left(\frac{1-\mu_{2}}{0.9}\right)\left(\frac{\mu_{2}}{0.1}\right)^{-1 / 4}\left(\frac{f_{\text {Edd }}}{0.1}\right)^{-1 / 4} \cdot
\end{aligned}
$$

For these fiducial parameters, the maximum detectable separation is smaller than the minimum allowed, and there is no range of separations for which a changing velocity-offset is detectable. Due to the weak scalings with parameters in Eq. 14, this is true for the majority of the plausible parameter space. Therefore, spectroscopic surveys which target changing BLR velocities are unlikely to detect signatures of binary AGN. If the binary mass ratio $q \lesssim 10^{-3}$, then a small range of separations with detectable changing-velocities opens up. Surveys which additionally target offset BLRs are more likely to be in the regime of Eq. 13, which makes searches even more challenging.

\subsection{Double-Peaked BLs}

For the BLs from both AGN to be observable, the binary orbital velocity must be larger than the combined characteristic widths of both BLs. As pointed out by, e.g., Chen et al. (1989); Eracleous \& Halpern (1994); Shen \& Loeb (2010b), this is generally in conflict with the need for each BLR to remain bound to its MBH and thus be closer-in than the binary orbit itself (see Fig. 1). Kinematically separated BL peaks from each AGN are thus unlikely to ever be observable. We can estimate the maximum orbital velocity that still preserves each BLR using Eq. 6,

$$
v_{\text {orb, } \max } \approx 1500 \mathrm{~km} \mathrm{~s}^{-1}\left(\frac{M}{10^{8} \mathrm{M}_{\odot}}\right)^{0.21}\left(\frac{\mu_{2}}{10^{-1}}\right)^{-0.13}\left(\frac{f_{\text {Edd }}}{10^{-1}}\right)^{-0.29},
$$

which is still a factor of two to ten lower than characteristic BL widths, $\sigma_{\text {BLR }} \approx 3 \times 10^{3}-10^{4} \mathrm{~km} \mathrm{~s}^{-1}$ (e.g., Stern \& Laor 2012).

Double-peaked BLs would seem very difficult to be produced from an AGN binary, unless BLs are able to be produced in binary systems from outside of each AGN's Hill radius-while still being bound to each AGN individually. Allowing the Hill sphere to partially impinge on the BLR modestly increases the maximum allowable orbital velocity, but likely also removes the lower-velocity portion of the BLs, making two distinct peaks more difficult to produce. A number of insightful reasons why most observed AGN with double-peaked BLs are unlikely to be in binaries are outlined in Eracleous \& Halpern (1994) and De Rosa et al. (2019). Notably, double-peaked BLs are much too common in single AGN (e.g. 20\% of radio-loud AGN; Eracleous \& Halpern 2003), and are well fit by single-AGN models.

\subsection{The sensitivity to velocity offsets}

The accuracy with which the centroid of a profile can be measured is particularly important in the pulsar timing and stellar/exoplanet communities. The sensitivity is often calculated using the CramérRao bound / Fischer information matrix or equivalently from a maximum likelihood treatment (e.g., Landman et al. 1982; Connes 1985; Butler et al. 1996; Lovis \& Fischer 2010; Beatty \& Gaudi 2015). Here we motivate the key scalings based on a simple example. Consider a triangular spectral feature (and perfectly matching model) of amplitude $(A)$ and full width at half maximum (FWHM: $W$ ). Fitting the model to the spectrum with a single pixel (i.e. resolution element) has a centroid uncertainty $\sigma_{i}$ due to both the pixel size $\left(\sigma_{\text {in }}\right)$ and the amplitude uncertainty (with root-mean-square amplitude $\sigma_{A}$ ). The amplitude uncertainty translates into a spectral uncertainty based on the feature's slope. This can be taken as independent and thus adds in quadrature. Defining the signal-to-noise ratio as $S_{\text {amp }} \equiv A / \sigma_{A}$, and identifying the number of resolution elements within the feature as $N_{\text {res }}=W / \sigma_{\text {in }}$, we can write,

$$
\sigma_{i}^{2}=\sigma_{\mathrm{in}}^{2}+\left(\frac{W}{A} \sigma_{A}\right)^{2} \approx \sigma_{\mathrm{in}}^{2}\left(\frac{N_{\mathrm{res}}}{S_{\mathrm{amp}}}\right)^{2}
$$

The approximation relies on the feature being wider than it is tall in terms of resolution, or equivalently that the SNR per pixel is small. We can convert from FWHM to Gaussian standard-deviation, and identify this with the characteristic width of the BL such that, $W=2(2 \ln 2)^{1 / 2} \sigma_{\text {BLR }}$. The combined accuracy of fitting with $N_{\text {res }}$ elements and $N_{\text {obs }}$ independent observations can then be written as,

$$
\begin{aligned}
\sigma_{v} & =(2 \ln 2)^{1 / 4} \frac{\left(\sigma_{\mathrm{BLR}} \sigma_{\mathrm{in}}\right)^{1 / 2}}{S} \\
& \approx 10^{2} \mathrm{~km} \mathrm{~s}^{-1} N_{\mathrm{obs}}^{-1 / 2}\left(\frac{S_{\mathrm{amp}}}{10^{1 / 2}}\right)^{-1}\left(\frac{\sigma_{\mathrm{BLR}}}{10^{4} \mathrm{~km} \mathrm{~s}^{-1}}\right)^{1 / 2}\left(\frac{\sigma_{\mathrm{in}}}{10 \mathrm{~km} \mathrm{~s}^{-1}}\right)^{1 / 2} .
\end{aligned}
$$

The derivation leading to Eq. 17 is simple and highly idealized, but in most respects it should represent an optimal sensitivity. BLs are of course not triangles, and in general are centrally-peaked-with the highest amplitude feature being much narrower, and contributing most of the SNR. The highest velocity material, on the other hand, which should best trace the offset AGN components, is much broader and contributes less to the SNR, which make the effective accuracy much lower. BL shapes are also known to be typically asymmetric and highly variable, both of which further degrade the accuracy of centroiding.

The best sensitivity is likely achieved by template matching, using a template constructed from independent observations to decrease the impact of uncorrelated temporal variations. Cordes \& Shannon (2010, Eq.A3), for example, give the minimum centroid error of template matching for pulsar pulses and find an expression matching the scalings of Eq. 17, and with a numerical pre-factor 
that differs by only $\approx 5 \%$. Their result is also identical to the traditional maximum-likelihood based estimates, for example in Landman et al. (1982, Eq. 4b), and should also be effectively very similar to the results of cross-correlation which are typically used for BLs. Beatty \& Gaudi (2015) show that variations to profile shape (e.g. Gaussian vs. Lorentzian, etc) lead to changes in the prefactor on the order of $\sim 10 \%$. Overall, this suggests that there is a fairly clear theoretical limit to centroid accuracy that is quite general and fairly insensitive to assumptions.

Here we have assumed an intrinsic BL velocity-width, $\sigma_{\text {BLR }} \approx$ $10^{4} \mathrm{~km} \mathrm{~s}^{-1}$. A somewhat lower value of $\approx 3 \times 10^{3} \mathrm{~km} \mathrm{~s}^{-1}$ is more representative of the bulk of observed AGN (e.g Stern \& Laor 2012). At the same time, the tails of BLs beyond the FWHM are produced closer to the AGN, and thus likely the best tracers of its motion. 'Instrumental broadening' in the literature is also typically much larger (e.g. $\gtrsim 100 \mathrm{~km} \mathrm{~s}^{-1}$ Barth et al. 2015) than our fiducial value of $\sigma_{\text {in }}=10 \mathrm{~km} \mathrm{~s}^{-1}$, though determining and comparing the most appropriate metric (i.e. systemic vs. per-pixel, at what SNR, etc) is not entirely obvious, and of course varies by instrument and methodology.

The preceding error analysis suggests that the accuracy to which AGN BL positions can be measured is $\sim 100 \mathrm{~km} \mathrm{~s}^{-1}$, at best. BLRs, however, are highly dynamical, and the ability to identify kinematic offsets as potential binary candidates also depends on the intrinsic stability of BL signals themselves. Velocity offsets of broad Balmer lines tend to vary ('jitter') over a few hundred $\mathrm{km} \mathrm{s}^{-1}$ over time intervals of $1-6$ months (Barth et al. 2015; Doan et al. 2019). Jitter amplitudes from Doan et al. (2019) are shown in Fig. A1, where the median jitter for red and blue components are $270 \mathrm{~km} / \mathrm{s}$ and $390 \mathrm{~km} / \mathrm{s}$ respectively.

A possible source of this jitter is that varying continuum emission reverberates off of BLR components whose Doppler factors vary asymmetrically with their time-delay (Blandford \& McKee 1982). For example, consider an AGN whose BLR is part of a fast outflow, oriented in a disk-like geometry that is inclined relative to the observer. If the continuum emission increases in brightness, the observer will first see it reverberate off of the nearer-side of the disk, which is blue-shifted from the outflow, and thus the early response will be a bluing of the line, followed later by a reddening. Barth et al. (2015) discuss numerous possible sources of jitter, including this "asymmetric reverberation" induced jitter, and find that it can produce variations of hundreds of $\mathrm{km} \mathrm{s}^{-1}$ on timescales comparable to the continuum variability.

AGN in MBH binary systems are expected to be intrinsically rare, with a fraction of all AGN at best $\lesssim 10^{-2}$ (e.g., Volonteri et al. 2009; Kelley et al. 2019b). Naively, a characteristic kinematic offset should be at least this rare $\left(10^{-2} \approx 2.3\right.$ Gaussian standarddeviations) in single AGN to hint at the presence of a binary. This implies a minimum velocity offset of $\gtrsim 230 \mathrm{~km} \mathrm{~s}^{-1}$ from Eq. 17 . The observational jitter measurements of Doan et al. (2019) imply a significantly higher empirical cutoff of $\gtrsim 600-800 \mathrm{~km} \mathrm{~s}^{-1}$, and the true high-jitter tails of the distribution are likely significantly larger than based on these $\approx$ dozen measurements.

Based on these considerations, taking $v_{\text {sens }} \sim 10^{3} \mathrm{~km} \mathrm{~s}^{-1}$ as a typical intrinsic sensitivity to kinematic offsets seems very reasonable, and is often used in the literature to select for offset systems (e.g., Eracleous et al. 2012b). Also, using this value for the sensitivity to changing velocity offsets ( $\Delta v_{\text {sens }}$ ) would seem similarly reasonable, whereas $\Delta v_{\text {sens }} \sim 10^{2} \mathrm{~km} \mathrm{~s}^{-1}$ would seem quite optimistic except, perhaps, in cases of very high SNR spectra, and AGN known to have BLs that are particularly stable. The latter, however, might itself substantially bias against binary AGN. The presence of a massive companion will generally seed and grow time-dependent variability in the accretion flow. While this is likely concentrated at timescales near the orbital period (e.g., Miranda et al. 2017), faster variations are certainly possible (e.g., Farris et al. 2014), for example if spiral density waves are excited in each AGN's circumsingle disk.

\section{KINEMATIC OFFSETS IN EVOLVING MBH BINARY POPULATIONS}

The binary parameter space that is accessible through kinematic offsets was shown in Fig. 3 and discussed above. The intrinsic distribution of binary parameters is biased towards low total masses and large separations (see, e.g., Kelley et al. 2017a). To account for the underlying parameter distributions, we use a simulated population of binary AGN and apply the selection criteria described in $\S 2$. The binary population is derived from the 'Illustris' cosmological, hydrodynamic simulations (Vogelsberger et al. 2013, 2014b; Torrey et al. 2014; Vogelsberger et al. 2014a; Genel et al. 2014; Sijacki et al. 2015), and evolved in post-processing using semianalytic models presented in (Kelley et al. 2017a,b). This produces a population of $\approx 10^{4}$ binaries, where each component mass is between $10^{6}$ and $\approx 10^{10} \mathrm{M}_{\odot}$.

Based on the cosmological evolution of the universe, we can extrapolate from this population in a finite volumeto an entire population of binaries in the observer's past light-cone. We employ kernel density-estimation (KDE), a type of non-parametric multidimensional parameter estimation, to infer the underlying distribution of binary properties, and then resample to the required tens to hundreds of millions of systems. The KDE approach (e.g., Scott \& Sain 2005) smooths each data point over a 'kernel' that fills in gaps between the data points, while still preserving the covariance and structure of the input. Resampling from a histogram is equivalent to a KDE with a top-hat kernel, at discrete locations. The KDE package we use is being generalized for use by the community Kelley (in prep.).

The accretion rate onto each component of the binaries is not resolved in Illustris (nor the structure of their disks or BLRs), although it does provide an accretion rate onto the combined system. How this overall accretion rate into post-merger galactic nuclei is partitioned between binary MBHs is unclear, particularly for the binary separations of interest here (see $\S 4$ ). Due to these uncertainties we continue to adopt a fixed Eddington factor To account for viewing angle effects for kinematic offsets $\left(v_{\text {off }}\right)$ and their shifts $(\Delta v)$, we use the expressions derived in Pflueger et al. (2018) for the probability of detecting a given offset $P_{v_{\text {off }}}\left(x \equiv v_{\text {sens }} / v_{\text {off }}\right)$ or shift $P_{\Delta v}\left(y \equiv \Delta v_{\text {sens }} / \Delta v\right)$,

$$
\begin{array}{r}
P_{v_{\mathrm{off}}}=1-\frac{2}{\pi}\left[\arcsin x+x \ln \left(\frac{1+\cos (\arcsin x)}{x}\right)\right], \\
P_{\Delta v}=\frac{2}{\pi}\left[\arccos y-y \ln \left(\frac{1+\sin (\arccos y)}{y}\right)\right] .
\end{array}
$$

Because $P_{v_{\text {off }}}$ and $P_{\Delta v}$ are strongly anti-correlated in viewing angle $(\S 2.3)$, we use the approximation that the combined probability $P_{v_{\text {off }} \Delta v}=P_{v_{\text {off }}} P_{\Delta v}$, which gives accurate results ${ }^{9}$.

9 Note that Pflueger et al. (2018) do provide an exact expression for $P_{v_{\text {off }} \Delta v}$, though it must be integrated numerically for each parameter combination. The approximation we use is typically accurate to $\sim 10 \%$ for individual systems, and better than $5 \%$ for the overall population. 

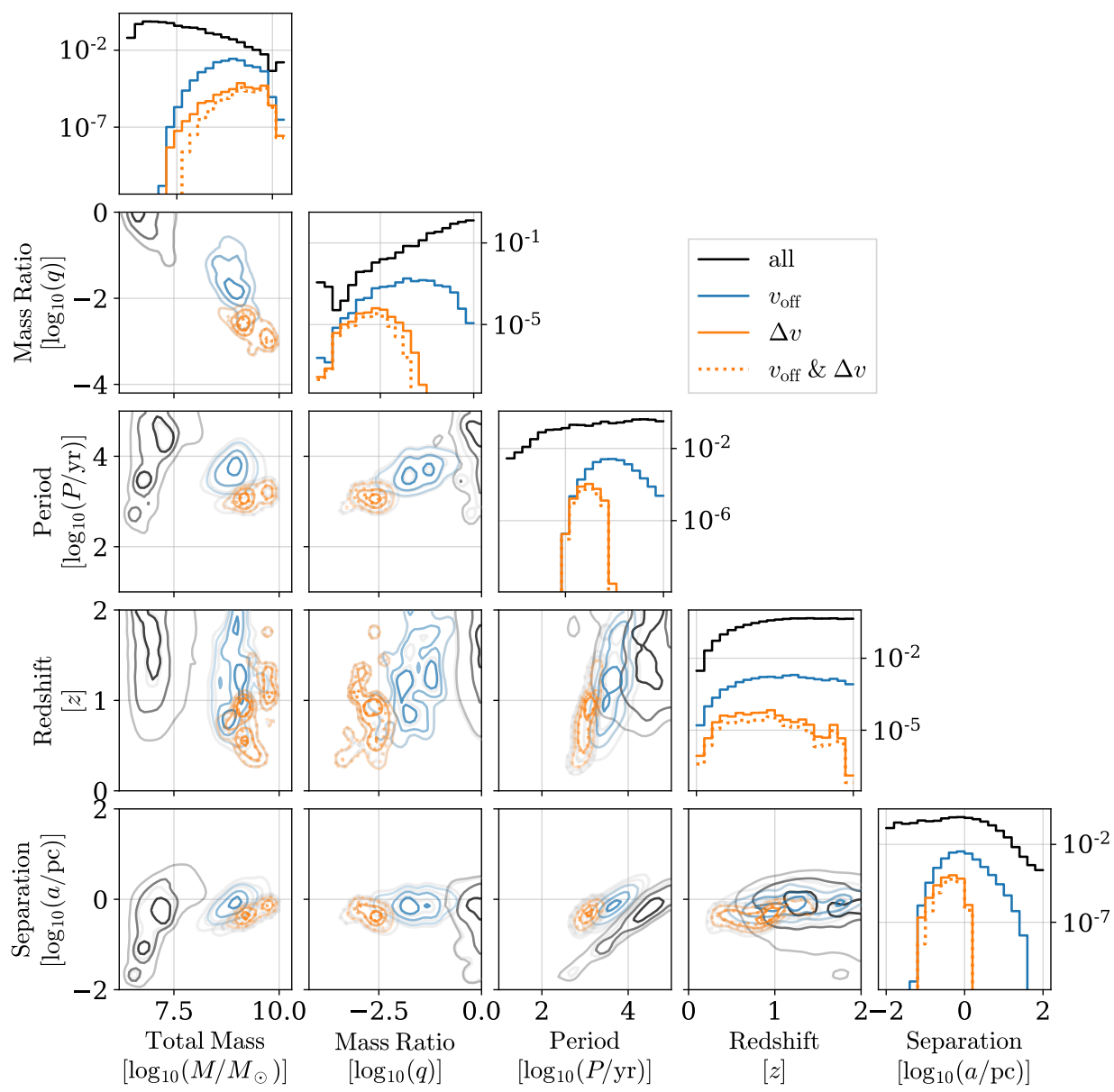

Figure 4. Distributions of parameters for all binaries (black) and those with a detectable kinematic signature in the secondary AGN (colors). Less than $10^{-7}$ of primaries are observable, which are not shown. Secondary AGN with observable velocity offsets ( $v_{\text {off }}$ ) are shown in blue assuming a sensitivity of $v_{\text {sens }}=10^{3} \mathrm{~km} \mathrm{~s}^{-1}$. Secondaries with observable changing velocities $(\Delta v)$ are shown in orange (solid) for a sensitivity $\Delta v_{\text {sens }}=10^{2} \mathrm{~km} \mathrm{~s} \mathrm{~s}^{-1}$, and observing baseline of $T_{\mathrm{obs}}=5 \mathrm{yr}$. Systems where both offsets and changes are detectable (orange, dotted) are mostly a uniform subset of the $\Delta v$ sample, and are almost indistinguishable in the $2 \mathrm{D}$ contour plots. An optical flux cutoff of $6 \times 10^{-14} \mathrm{erg} / \mathrm{s} / \mathrm{cm}^{2}(\mathrm{AB} \mathrm{Mag} \approx 21)$ is also imposed, where all AGN are assumed to be accreting at an Eddington fraction of 0.1 .

The distribution of binary parameters are shown in Fig. 4 for all binaries and those with detectable kinematic signatures. The populations are compared for all binaries (black), those with a detectable kinematic offset $\left(v_{\text {off }}\right.$, blue), changing offset $(\Delta v$, orange solid), and both (orange dashed). Because, generally, $P_{\Delta v} \ll P_{v_{\text {off }}}$, half $(52 \%)$ of systems with a detectable changing-offset also have offsets $v_{\text {off }}>v_{\text {sens }}$. All binaries, and those with detectable $v_{\text {off }}$, and $\Delta v$, are each concentrated in different regions of parameters space. All binaries tend to have low total masses, high-mass ratios ${ }^{10}$, and long orbital periods where their residence times $a /(d a / d t)$ are longest. The decline in systems with $a \gtrsim 10 \mathrm{pc}$ is due to systems not being gravitationally bound at larger separations. All detectable systems are biased towards the largest total masses, and more extreme mass-ratios, which produce the largest orbital velocities in the secondary AGN. For the same reason, detectable systems prefer smaller separations — but below $\sim 0.1 \mathrm{pc}$, the shrinking Hill radii

10 Note that this is partially a selection effect because we only include MBH masses $M>10^{6} \mathrm{M}_{\odot}$, and thus the dominant number of binaries with total-masses near $M \sim 10^{6} \mathrm{M}_{\odot}$ can only have near-equal mass ratios. begin to exclude BLRs. Only one in roughly every four million binaries has a detectable primary offset, and these never have a detectable changing offset. None of our binaries have double-peaked BLs with offsets in both the primary and secondary AGN. We therefore focus our analysis on systems with a detectable secondary.

The fraction of binaries that are kinematically detectable are plotted in Fig. 5 for a subset of parameters. Overall, $5 \times 10^{-3}$ of binaries have a secondary AGN with detectable $v_{\text {off }}>10^{3} \mathrm{~km} \mathrm{~s}^{-1}$, and only $3 \times 10^{-4}$ with $\Delta v>10^{2} \mathrm{~km} \mathrm{~s}^{-1}$. Parametrically, systems with detectable $\Delta v$ are a fairly uniform subset of those with detectable $v_{\text {off. }}$. The $\Delta v$ and $v_{\text {off }}$ are necessarily anti-correlated in orbital phase (\$2.3). For this reason only half of $\Delta v$ detectable systems also have a detectable $v_{\text {off }}$, at $1.4 \times 10^{-4}$ of all binaries. We have also tried including the scatter from observational scaling relationships in our calculations, in particular the scatter in $M_{\mathrm{BH}}-\sigma_{\star}$ from McConnell \& Ma (2013) and BLâĂŞ $H_{\beta}$ radiusâĂŞluminosity from Bentz et al. (2013). The changes to the plotted histograms are imperceptible, and the overall detection rates are hardly changed: systems with detectable $\Delta v$ go from $2.7 \times 10^{-4}$ to $2.8 \times 10^{-4}$, and those with both $\Delta v$ and $v_{\text {off }}$ go from $1.4 \times 10^{-4}$ to $1.5 \times 10^{-4}$.

Our binary evolution models take into account the mass ra- 


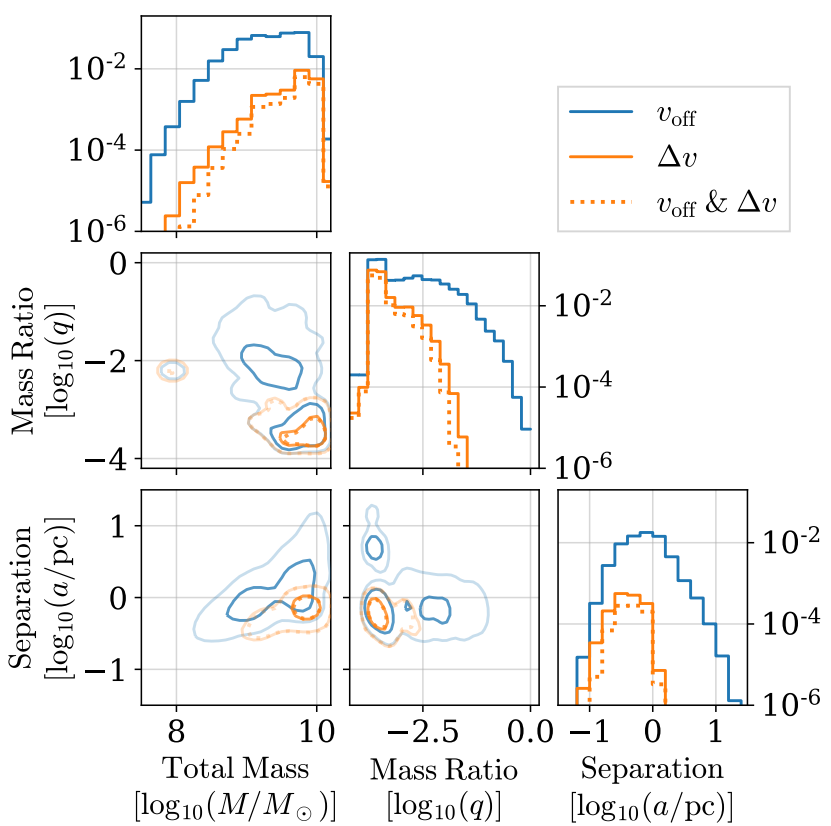

Figure 5. Fraction of binary AGN with detectable kinematic signatures from the secondary. The same parameters are used as in Fig. 4: $v_{\text {sens }}=10^{3} \mathrm{~km} \mathrm{~s}^{-1}$, observing baseline $T_{\text {obs }}=5 \mathrm{yr}, \Delta v_{\text {sens }}=10^{2} \mathrm{~km} \mathrm{~s}^{-1}$, and $F_{\text {opt }}>6 \times 10^{-14} \mathrm{erg} / \mathrm{s} / \mathrm{cm}^{2}\left(M_{\mathrm{AB}} \approx 21\right)$. Overall, $0.49 \%$ of secondaries have a detectable offset and only $0.027 \%$ have detectable changes in velocity. Both kinematic signatures are simultaneously detectable in $0.014 \%$ of systems. Distributions of binary parameters for detectable systems are listed in Table A1.

tios, both of the MBHs and their host galaxies, in all stages of their evolution. Still, the dynamics of more extreme mass-ratio systems are even more uncertain than that of MBHBs overall. Our analysis also does not take into account that the secondary AGN, which always produced the detectable kinematic offsets, must be discernible behind the emission of the primary, which may be much brighter. For these reasons we caution that the number of detectable extreme mass-ratio systems may be overestimated here.

As apparent in Fig. 4, detectable binary parameters are only very weakly dependent on redshift. The $\mathrm{H} \beta \mathrm{BL}$ is one of the most commonly used to search for binary candidates, but is only observable in SDSS out to a redshift of $\approx 0.7$; , though MgII and CIV are useful at larger redshifts. In Table A1 we include tabulated quantiles for detectable systems at low to moderate redshifts: $z<0.7$.

\section{DISCUSSION \& CONCLUSIONS}

Based on empirical scaling relations in AGN BL properties, and simple physical considerations, we have shown that kinematically offset AGN are detectable in only a narrow range of parameter space. BL regions from relatively bright AGN are observed to have characteristic radii on the order of 0.1 pc (e.g., Kaspi et al. 2007; Bentz et al. 2013), suggesting that binary separations must be at least this wide for their BLs to remain observable, and to trace the motion of each AGN component. At binary separations larger than $\sim 1 \mathrm{pc}$, orbital velocities become too small to reliably detect. For the same reason, binaries of total mass much less than $\approx 10^{8} \mathrm{M}_{\odot}$ are rarely detectable. Even if we have underestimated the sensitivity of spectroscopic surveys to kinematic offsets, the accretion disk radii corresponding to BLRs in lower mass systems may be suscep- tible to gravitational instability. The dynamical stability of disks, especially in the case of circumbinary disks, however, is highly uncertain.

Requiring a minimum binary separation implies that the shortest orbital periods of kinematically detectable systems is optimistically hundreds of $y r$, with periods of $10^{3}-10^{4} \mathrm{yr}$ being typical. While binary candidates from AGN with photometric, periodicallyvariability can be filtered to some degree by waiting for numerous complete cycles (e.g., Sesana et al. 2018), likely this cannot be done for kinematic candidates.

Much of our analysis hinges on the premise that BLs are only observable if produced at the measured, characteristic radii of BLRs. While these measurements span a wide range of AGN parameters (e.g. redshift and luminosity; Kaspi et al. 2007), the total number of systems by which they are calibrated is still relatively small and may suffer from selection biases. How BLs are formed in more complex environments, such as MBH binaries and their circumbinary- and circumsingle- disks are only beginning to be explored in detail (e.g., Bogdanović et al. 2008; Shen \& Loeb 2010a; Nguyen \& Bogdanović 2016; Nguyen et al. 2019b). Studies such as these are starting to show how intricate the BLR environment can be around binary systems. In our analysis, and all others on binary AGN BLs to our knowledge, feedback processes such as energetic outflows and jets have been entirely neglected. These processes could not only alter but even disrupt a companion's BLR (either dynamically or radiatively).

The same considerations apply to dusty tori immediately outside of BLRs. The absence of dusty tori may be characteristic for AGN with binary companions (see middle-panel of Fig. 1), though this is highly speculative. If it is the case, it may be detectable through an infrared deficit. Obscuration of the BLR for viewing angles near the plane of the AGN disk, one of the hallmarks of AGN unification (e.g., Antonucci \& Miller 1985; Antonucci 1993; Urry \& Padovani 1995; Urry 2004; Nenkova et al. 2008; but, cf. Penston \& Perez 1984; DiPompeo et al. 2017), has also been neglected in our analysis. Dusty torus truncation may remediate some obscuration effects, but not if they are instead produced by high columndensities from dusty outflows (e.g., Konigl \& Kartje 1994; Elitzur \& Shlosman 2006), or time-dependent but more isotropic obscurers. Galaxy mergers also trigger increases in AGN obscuration (e.g., Hopkins et al. 2005; Koss et al. 2016; Ricci et al. 2017; Blecha et al. 2018; Pfeifle et al. 2019), though how the timing and duration of increased obscuration compares to MBHB inspiral times and the lifetimes of kinematically-detectable AGN is unclear.

Our analysis is sensitive to the fundamental accuracy by which BL centroids can be measured. Based on highly-simplified profile fitting considerations, we find that accuracies are likely at best $\sim 100 \mathrm{~km} \mathrm{~s}^{-1}$. BLs are known to be asymmetric and highly variable, where studies have shown that their velocities can shift by many hundreds of $\mathrm{km} \mathrm{s}^{-1}$ on month to year timescales (Barth et al. 2015; Doan et al. 2019). Based on these considerations, we use a fiducial sensitivity to velocity offsets of $v_{\text {sens }}=10^{3} \mathrm{~km} \mathrm{~s}^{-1}$, and what we consider to be a very optimistic sensitivity to changing velocities of $\Delta v_{\text {sens }}=10^{2} \mathrm{~km} \mathrm{~s}^{-1}$. These considerations do not account for possible contamination of offset lines produced by post-merger, recoiling AGN (e.g., Blecha et al. 2016; Sayeb et al. prep); but ultimately, in that case, it would still mark the presence of a past binary. The amplitude of velocity shifts from Brownian motion of singleAGN are expected to be very small (e.g., Merritt 2001), at least in 
relatively massive galaxies ${ }^{11}$, and thus should not be an important confusion or noise source.

We apply our observability criteria to a population of Illustris $\mathrm{MBH}$ binaries with masses between $\approx 10^{6}-10^{10} \mathrm{M}_{\odot}$, that are evolved from galaxy merger at kpc-scale separations down to eventual coalescence (Kelley et al. 2017a,b). Using the fiducial sensitivities discussed above, and assuming a universal Eddington fraction of 0.1 , we find that the secondary AGN should have a detectable kinematic offset in $0.5 \%$ of binaries. Changes in the BL velocity are only detectable in $0.03 \%$ of systems, with roughly half of those also having a detectable offset from the rest frame. Detectable systems have binary separations near and just below $1 \mathrm{pc}$, total masses $\gtrsim 10^{9} \mathrm{M}_{\odot}$, and extreme mass-ratios, typically with $q \lesssim 10^{-2}$. We caution that the evolution of these extreme mass-ratio systems is especially uncertain. For example, if accretion strongly favors the secondary, then mass ratios may evolve noticeably during inspiral (Siwek et al. 2020) which may decrease the occurrence rate of binary AGN with $q \lesssim 0.1$. Given our model assumptions, and the overall fraction of AGN in binaries being $10^{-2}-10^{-3}$ (Volonteri et al. 2009; Kelley et al. 2019b), less than one in $10^{4}$ AGN should have detectable kinematic signatures from binary motion. These results are not significantly effected by the scatter in observed scaling relationships.

One of the key differences between this analysis and previous ones is the consideration of the characteristic radii of optical BLRs: if the observed scalings hold in binaries, then the binary separation must be large enough that the BLR remains within the Hill sphere of each component AGN. This requirement is in tension with the need for small orbital separations to produce detectably large velocity shifts. The parameters used in this study are motivated primarily by studies of the $H_{\beta}$ line. Other optical BLs (e.g. MgII, CIV), show very similar characteristics (e.g., Homayouni et al. 2020), and the same overall conclusions likely apply. X-ray lines, like Fe$\mathrm{K}_{\alpha}$, which are produced in the immediate vicinities of MBHs, can have significantly larger Doppler shifts, are unaffected by truncation from the companion $\mathrm{MBH}$, and may tend to be brighter in the secondary AGN. Especially in light of proposed X-ray missions such as Athena (Barcons et al. 2017), Lynx (The Lynx Team 2018), and XRISM (XRISM Science Team 2020), prospects for detecting MBH binaries through X-Ray observations (e.g., Yu \& Lu 2001; McKernan et al. 2013; McKernan \& Ford 2015; Haiman 2017) should continue to be explored.

We find that the primary AGN in binaries are effectively undetectable. We do not account for whether or not the secondary AGN, which are significantly lower in mass than the primaries in detectable systems, are able to produce BLs bright enough to outshine the primary. Many studies have found that in circumbinary systems, accretion preferentially favors the secondary (Artymowicz \& Lubow 1994; Gould \& Rix 2000; Farris et al. 2014). As pointed out in Pflueger et al. (2018), this does not necessarily mean the secondary will also have a brighter BL, as the Hill sphere (and thus secondary accretion disk and BLR) is smaller than the primary. Indeed, the analysis in Nguyen et al. (2019a,b) suggests that even if the secondary is accreting preferentially, the primary dominates the $H_{\beta}$ line, making detection of kinematic signatures in the secondary far more difficult.

A number of reasons why double-peaked BLs are unlikely

11 though wandering and spatially-offset MBH are a rapidly developing field of research, e.g., Tremmel et al. (2018) and Reines et al. (2020, cf. Eftekhari et al. 2020) to be produced by the two BLs of a binary AGN are outlined by Eracleous et al. (and others), particularly in Eracleous \& Halpern (1994). Ultimately, it is very difficult to construct binary parameters in which the difference in orbital velocities between components can reach the width of the primary's BL. We find no systems in our population where this occurs. Theoretical considerations of disk alignment suggest that circumsingle disks (and thus possibly BLRs) may tend to be coplanar with the binary orbit (e.g., Artymowicz \& Lubow 1994; Ivanov et al. 1999; Bogdanović et al. 2007; Dotti et al. 2010; Miller \& Krolik 2013). If this is the case, and if a dusty torus is still present in the system, obscuration could be an even worse issue. If both components of the binary contribute noticeably to a combined BL, then the primary may not only mask the brightness of the secondary's BL, but its opposing velocity may negate some of the apparent velocity shift.

Unfortunately, our analysis has yielded a fairly pessimistic forecast for the detectability of binary MBHs through kinematic offsets in AGN optical spectra. Our ability to identify binary AGN in this way rests crucially on being able to better characterize the intrinsic asymmetry and variability of BLs and their production regions. In addition to existing data from reverberation mapping campaigns, a larger sample of AGN with multi-epoch spectroscopy, over a variety of timescales, is needed to understand what produces the 'jitter' in AGN BLRs. Those variations, especially if they are produced in response to continuum fluctuations, may be able to be characterized and modeled-out when searching for binary signatures. A campaign like the BH-Mapper (part of SDSS-V, Kollmeier et al. 2017) could provide invaluable constraints, and significantly increase the population of AGN required to plausibly identify the signposts of binarity which appear to be so rare.

\section{ACKNOWLEDGMENTS}

I am very thankful to Claude-André Fauche-Gigueré, Diego Muñoz and Jonathan Stern for frequent consultations and advise. Laura Blecha, Tamara Bogdanović, Claude-André FaucheGigueré, Jessie Runnoe, and Jonathan Stern provided thorough and invaluable feedback on an early draft. I also thank Maria Charisi and Joseph Simon for encouraging and stimulating conversations on the subject of kinematic binary detections.

This research made use of Astropy, a community-developed core Python package for Astronomy (Astropy Collaboration et al. 2013), in addition to SciPy (Jones et al. 2001), ipython (Pérez \& Granger 2007), jupyter notebook (Kluyver et al. 2016), NumPy (van der Walt et al. 2011) \& SymPy (Meurer et al. 2017). All figures were generated using matplotlib (Hunter 2007). Kernel density estimation was performed using the $\mathrm{k}$ alepy package (github.com/lzkelley/kalepy) (Kelley prep).

The Illustris data is available online at www.illustrisproject.org (Nelson et al. 2015), and Illustris-TNG data at www.tng-project.org (Nelson et al. 2019).

\section{REFERENCES}

Abraham Z., 2000, Astron. Astrophys., 355, 915

Agudo I., Marscher A. P., Jorstad S. G., Gómez J. L., Perucho M., Piner B. G., Rioja M., Dodson R., 2012, Astrophys. J., 747, 63 Antonucci R., 1993, Annu. Rev. Astron. Astrophys., 31, 473 Antonucci R. R. J., Miller J. S., 1985, Astrophys. J., 297, 621 Artymowicz P., Lubow S. H., 1994, Astrophys. J., 421, 651 
Astropy Collaboration et al., 2013, Astron. Astrophys., 558, A33

Bansal K., Taylor G. B., Peck A. B., Zavala R. T., Romani R. W., 2017, Astrophys. J., 843, 14

Barcons X., et al., 2017, Astronomische Nachrichten, 338, 153

Barnes J. E., Hernquist L. E., 1991, Astrophys. J. Lett., 370, L65

Barnes J. E., Hernquist L., 1992, Annu. Rev. Astron. Astrophys., 30, 705

Barth A. J., et al., 2015, The Astrophysical Journal Supplement Series, 217, 26

Barvainis R., 1987, Astrophys. J., 320, 537

Baskin A., Laor A., 2018, Mon. Not. R. Astron. Soc., 474, 1970

Baskin A., Laor A., Stern J., 2014, Mon. Not. R. Astron. Soc., 438, 604

Beatty T. G., Gaudi B. S., 2015, PASP, 127, 1240

Begelman M. C., Blandford R. D., Rees M. J., 1980, Nature, 287, 307

Bennert N., Canalizo G., Jungwiert B., Stockton A., Schweizer F., Peng C. Y., Lacy M., 2008, Astrophys. J., 677, 846

Bentz M. C., et al., 2013, Astrophys. J., 767, 149

Blandford R. D., McKee C. F., 1982, Astrophys. J., 255, 419

Blecha L., et al., 2016, Mon. Not. R. Astron. Soc., 456, 961

Blecha L., Snyder G. F., Satyapal S., Ellison S. L., 2018, Mon. Not. R. Astron. Soc., 478, 3056

Blumenthal G. R., Faber S. M., Primack J. R., Rees M. J., 1984, Nature, 311,517

Bogdanović T., 2015, in Gravitational Wave Astrophysics. p. 103 (arXiv: 1406.5193), doi:10.1007/978-3-319-10488-1_9

Bogdanović T., Reynolds C. S., Miller M. C., 2007, Astrophys. J. Lett., 661, L147

Bogdanović T., Smith B. D., Sigurdsson S., Eracleous M., 2008, Astrophys. J., Suppl. Ser., 174, 455

Bon E., et al., 2012, Astrophys. J., 759, 118

Boroson T. A., Lauer T. R., 2009, Nature, 458, 53

Britzen S., et al., 2018, Mon. Not. R. Astron. Soc., 478, 3199

Brotherton M. S., Runnoe J. C., Shang Z., DiPompeo M. A., 2015, Mon. Not. R. Astron. Soc., 451, 1290

Butler R. P., Marcy G. W., Williams E., McCarthy C., Dosanjh P., Vogt S. S., 1996, PASP, 108, 500

Chen K., Halpern J. P., Filippenko A. V., 1989, Astrophys. J., 339, 742

Clavel J., Wamsteker W., Glass I. S., 1989, Astrophys. J., 337, 236

Coffey D., et al., 2019, Astron. Astrophys., 625, A123

Comerford J. M., Greene J. E., 2014, Astrophys. J., 789, 112

Comerford J. M., et al., 2009, Astrophys. J., 698, 956

Comerford J. M., Gerke B. F., Stern D., Cooper M. C., Weiner B. J., Newman J. A., Madsen K., Barrows R. S., 2012, Astrophys. J., 753, 42

Connes P., 1985, Ap\&SS, 110, 211

Cordes J. M., Shannon R. M., 2010, arXiv e-prints, p. arXiv:1010.3785

Davis M., Efstathiou G., Frenk C. S., White S. D. M., 1985, Astrophys. J., 292, 371

De Rosa A., et al., 2019, New Astron. Rev., 86, 101525

Decarli R., Dotti M., Fumagalli M., Tsalmantza P., Montuori C., Lusso E., Hogg D. W., Prochaska J. X., 2013, Mon. Not. R. Astron. Soc., 433, 1492

Detweiler S., 1979, Astrophys. J., 234, 1100

DiPompeo M. A., Hickox R. C., Eftekharzadeh S., Myers A. D., 2017, Mon. Not. R. Astron. Soc., 469, 4630

Doan A., et al., 2019, ] 10.1093/mnras/stz2705, 18, 1

Doan A., Eracleous M., Runnoe J. C., Liu J., Mathes G., Flohic H. M. L. G., 2020, Mon. Not. R. Astron. Soc., 491, 1104

Dotti M., Montuori C., Decarli R., Volonteri M., Colpi M., Haardt F., 2009, Mon. Not. R. Astron. Soc., 398, L73

Dotti M., Volonteri M., Perego A., Colpi M., Ruszkowski M., Haardt F., 2010, Mon. Not. R. Astron. Soc., 402, 682

Du P., et al., 2018, Astrophys. J., 869, 142

Eftekhari T., Berger E., Margalit B., Metzger B. D., Williams P. K. G., 2020, arXiv e-prints, p. arXiv:2001.02688

Eggleton P. P., 1983, Astrophys. J., 268, 368

Elitzur M., Shlosman I., 2006, Astrophys. J. Lett., 648, L101

Eracleous M., Halpern J. P., 1994, Astrophys. J., Suppl. Ser., 90, 1

Eracleous M., Halpern J. P., 2003, Astrophys. J., 599, 886
Eracleous M., Livio M., Halpern J. P., Storchi-Bergmann T., 1995, Astrophys. J., 438, 610

Eracleous M., Halpern J. P., M. Gilbert A., Newman J. A., Filippenko A. V., 1997a, Astrophys. J., 490, 216

Eracleous M., Halpern J. P., M. Gilbert A., Newman J. A., Filippenko A. V., 1997b, Astrophys. J., 490, 216

Eracleous M., Lewis K. T., Flohic H. M. L. G., 2009, New Astron. Rev., 53, 133

Eracleous M., Boroson T. A., Halpern J. P., Liu J., 2012a, Astrophys. J., Suppl. Ser., 201, 23

Eracleous M., Boroson T. A., Halpern J. P., Liu J., 2012b, Astrophys. J., Suppl. Ser., 201, 23

Farris B. D., Duffell P., MacFadyen A. I., Haiman Z., 2014, Astrophys. J., 783,134

Foster R. S., Backer D. C., 1990, Astrophys. J., 361, 300

Gaskell C. M., 1983a, in Swings J.-P., ed., Liege International Astrophysical Colloquia Vol. 24, Liege International Astrophysical Colloquia. pp $473-477$

Gaskell C. M., 1983b, Nature, 304, 212

Gaskell C. M., 1996, Astrophys. J. Lett., 464, L107

Genel S., et al., 2014, Mon. Not. R. Astron. Soc., 445, 175

Gould A., Rix H.-W., 2000, Astrophys. J. Lett., 532, L29

Goulding A. D., et al., 2018, PASJ, 70, S37

Guo Q., White S. D. M., 2008, Mon. Not. R. Astron. Soc., 384, 2

Guo H., Liu X., Shen Y., Loeb A., Monroe T., Prochaska J. X., 2019, Mon. Not. R. Astron. Soc., 482, 3288

Haiman Z., 2017, Phys. Rev. D, 96, 023004

Haiman Z., Kocsis B., Menou K., 2009, Astrophys. J., 700, 1952

Halpern J. P., Filippenko A. V., 1988, Nature, 331, 46

Hellings R. W., Downs G. S., 1983, Astrophys. J. Lett., 265, L39

Homayouni Y., et al., 2020, arXiv e-prints, p. arXiv:2005.03663

Hopkins P. F., Hernquist L., Martini P., Cox T. J., Robertson B., Di Matteo T., Springel V., 2005, Astrophys. J. Lett., 625, L71

Hunter J. D., 2007, Computing In Science \& Engineering, 9, 90

Ivanov P. B., Papaloizou J. C. B., Polnarev A. G., 1999, Mon. Not. R. Astron. Soc., 307, 79

Jones E., Oliphant T., Peterson P., et al., 2001, SciPy: Open source scientific tools for Python, Link

Ju W., Greene J. E., Rafikov R. R., Bickerton S. J., Badenes C., 2013, Astrophys. J., 777, 44

Kaspi S., Smith P. S., Maoz D., Netzer H., Jannuzi B. T., 1996, Astrophys. J. Lett., 471, L75

Kaspi S., Smith P. S., Netzer H., Maoz D., Jannuzi B. T., Giveon U., 2000, Astrophys. J., 533, 631

Kaspi S., Brandt W. N., Maoz D., Netzer H., Schneider D. P., Shemmer O., 2007, Astrophys. J., 659, 997

Kelley L. Z., in prep

Kelley L. Z., Blecha L., Hernquist L., 2017a, Mon. Not. R. Astron. Soc., 464, 3131

Kelley L. Z., Blecha L., Hernquist L., Sesana A., Taylor S. R., 2017b, Mon. Not. R. Astron. Soc., 471, 4508

Kelley L. Z., Blecha L., Hernquist L., Sesana A., Taylor S. R., 2018, Mon. Not. R. Astron. Soc., 477, 964

Kelley L., et al., 2019a, BAAS, 51, 490

Kelley L. Z., Haiman Z., Sesana A., Hernquist L., 2019b, Mon. Not. R. Astron. Soc., 485, 1579

Kluyver T., et al., 2016, in Loizides F., Schmidt B., eds, Positioning and Power in Academic Publishing: Players, Agents and Agendas. pp 87 90

Kollmeier J. A., et al., 2017, arXiv e-prints, p. arXiv:1711.03234

Komberg B. V., 1968, Soviet Astronomy, 11, 727

Komossa S., 2006, Mem. Societa Astronomica Italiana, 77, 733

Konigl A., Kartje J. F., 1994, Astrophys. J., 434, 446

Koratkar A. P., Gaskell C. M., 1991, Astrophys. J. Lett., 370, L61

Kormendy J., Richstone D., 1995, Annu. Rev. Astron. Astrophys., 33, 581

Koshida S., et al., 2014, Astrophys. J., 788, 159

Koss M., Mushotzky R., Treister E., Veilleux S., Vasudevan R., Trippe M., 2012, Astrophys. J. Lett., 746, L22 
Koss M. J., et al., 2016, Astrophys. J., 825, 85

Kovačević A. B., Wang J.-M., Popović L. Č., 2020, Astron. Astrophys., 635, A1

Lacey C., Cole S., 1993, Mon. Not. R. Astron. Soc., 262, 627

Laine S., et al., 2020, Astrophys. J. Lett., 894, L1

Landman D. A., Roussel-Dupre R., Tanigawa G., 1982, Astrophys. J., 261, 732

Lin D. N. C., Papaloizou J., 1979, Mon. Not. R. Astron. Soc., 186, 799

Liu X., Shen Y., Bian F., Loeb A., Tremaine S., 2014, Astrophys. J., 789, 140

Liu J., Eracleous M., Halpern J. P., 2016, Astrophys. J., 817, 42

Lotz J. M., Jonsson P., Cox T. J., Croton D., Primack J. R., Somerville R. S., Stewart K., 2011, Astrophys. J., 742, 103

Lovis C., Fischer D., 2010, Radial Velocity Techniques for Exoplanets. pp 27-53

Lu W.-J., Lin Y.-R., 2019, Astrophys. J., 887, 178

Magorrian J., et al., 1998, Astron. J., 115, 2285

Martin R. G., Lubow S. H., 2011, Mon. Not. R. Astron. Soc., 413, 1447

McConnell N. J., Ma C.-P., 2013, Astrophys. J., 764, 184

McKernan B., Ford K. E. S., 2015, Mon. Not. R. Astron. Soc., 452, L1

McKernan B., Ford K. E. S., Kocsis B., Haiman Z., 2013, Mon. Not. R. Astron. Soc., 432, 1468

Merritt D., 2001, Astrophys. J., 556, 245

Merritt D., Milosavljević M., 2005, Living Reviews in Relativity, 8

Meurer A., et al., 2017, PeerJ Computer Science, 3, e103

Miller M. C., Krolik J. H., 2013, Astrophys. J., 774, 43

Milosavljević M., Merritt D., 2003, Astrophys. J., 596, 860

Mingarelli C. M. F., et al., 2017, Nature Astronomy, 1, 886

Miranda R., Lai D., 2015, Mon. Not. R. Astron. Soc., 452, 2396

Miranda R., Muñoz D. J., Lai D., 2017, Mon. Not. R. Astron. Soc., 466, 1170

Montuori C., Dotti M., Colpi M., Decarli R., Haardt F., 2011, Mon. Not. R. Astron. Soc., 412, 26

Montuori C., Dotti M., Haardt F., Colpi M., Decarli R., 2012, Mon. Not. R. Astron. Soc., 425, 1633

Nelson D., et al., 2015, Astronomy and Computing, 13, 12

Nelson D., et al., 2019, Computational Astrophysics and Cosmology, 6, 2

Nenkova M., Sirocky M. M., Nikutta R., Ivezić Ž., Elitzur M., 2008, Astrophys. J., 685, 160

Netzer H., Laor A., 1993, Astrophys. J. Lett., 404, L51

Newman A. B., Ellis R. S., Bundy K., Treu T., 2012, Astrophys. J., 746, 162

Nguyen K., Bogdanović T., 2016, Astrophys. J., 828, 68

Nguyen K., Bogdanovic T., Runnoe J. C., Eracleous M., Sigurdsson S., Boroson T., 2019a, arXiv e-prints, p. arXiv:1908.01799

Nguyen K., Bogdanović T., Runnoe J. C., Eracleous M., Sigurdsson S., Boroson T., 2019b, Astrophys. J., 870, 16

Paczynski B., 1977, Astrophys. J., 216, 822

Pancoast A., Brewer B. J., Treu T., Park D., Barth A. J., Bentz M. C., Woo J.-H., 2014, Mon. Not. R. Astron. Soc., 445, 3073

Penston M. V., Perez E., 1984, Mon. Not. R. Astron. Soc., 211, 33P

Pérez F., Granger B., 2007, Computing in Science Engineering, 9, 21

Pfeifle R. W., et al., 2019, Astrophys. J., 875, 117

Pflueger B. J., Nguyen K., Bogdanović T., Eracleous M., Runnoe J. C., Sigurdsson S., Boroson T., 2018, Astrophys. J., 861, 59

Pichardo B., Sparke L. S., Aguilar L. A., 2005, Mon. Not. R. Astron. Soc., 359,521

Popović L. Č., 2012, New Astron. Rev., 56, 74

Rajagopal M., Romani R. W., 1995, Astrophys. J., 446, 543

Reines A. E., Condon J. J., Darling J., Greene J. E., 2020, Astrophys. J., 888,36

Ricci C., et al., 2017, Mon. Not. R. Astron. Soc., 468, 1273

Rodriguez-Gomez V., et al., 2015, Mon. Not. R. Astron. Soc., 449, 49

Rodriguez C., Taylor G. B., Zavala R. T., Peck A. B., Pollack L. K., Romani R. W., 2006, Astrophys. J., 646, 49

Rosado P. A., Sesana A., Gair J., 2015, Mon. Not. R. Astron. Soc., 451, 2417
Runnoe J. C., Brotherton M. S., Shang Z., 2012, Mon. Not. R. Astron. Soc., 422,478

Runnoe J. C., et al., 2015, Astrophys. J., Suppl. Ser., 221, 7

Runnoe J. C., et al., 2017, Mon. Not. R. Astron. Soc., 468, 1683

Sanders D. B., Soifer B. T., Elias J. H., Madore B. F., Matthews K., Neugebauer G., Scoville N. Z., 1988, Astrophys. J., 325, 74

Sayeb M., Blecha L., Kelley L. Z., in prep

Sazhin M. V., 1978, Soviet Astronomy, 22, 36

Scott D. W., Sain S. R., 2005, in Rao C., Wegman E., Solka J., eds, Handbook of Statistics, Vol. 24, Data Mining and Data Visualization. Elsevier, pp 229 - 261, doi:https://doi.org/10.1016/S0169-7161(04)240093, Link

Sesana A., Haardt F., Madau P., Volonteri M., 2004, Astrophys. J., 611, 623 Sesana A., Haiman Z., Kocsis B., Kelley L. Z., 2018, Astrophys. J., 856, 42 Shen Y., Loeb A., 2010a, Astrophys. J., 725, 249

Shen Y., Loeb A., 2010b, Astrophys. J., 725, 249

Shen Y., Liu X., Greene J. E., Strauss M. A., 2011, Astrophys. J., 735, 48

Shen Y., Liu X., Loeb A., Tremaine S., 2013, Astrophys. J., 775, 49

Sijacki D., Vogelsberger M., Genel S., Springel V., Torrey P., Snyder G. F., Nelson D., Hernquist L., 2015, Mon. Not. R. Astron. Soc., 452, 575

Sillanpää A., Haarala S., Valtonen M. J., Sundelius B., Byrd G. G., 1988, Astrophys. J., 325, 628

Siwek M. S., Kelley L. Z., Hernquist L., 2020, arXiv e-prints, p. arXiv:2005.09010

Soltan A., 1982, Mon. Not. R. Astron. Soc., 200, 115

Stern J., Laor A., 2012, Mon. Not. R. Astron. Soc., 423, 600

Stockton A., Farnham T., 1991, Astrophys. J., 371, 525

Storchi-Bergmann T., et al., 2003, Astrophys. J., 598, 956

Strateva I. V., et al., 2003, Astron. J., 126, 1720

Suganuma M., et al., 2006, Astrophys. J., 639, 46

Taylor S. R., Vallisneri M., Ellis J. A., Mingarelli C. M. F., Lazio T. J. W., van Haasteren R., 2016, Astrophys. J. Lett., 819, L6

The Lynx Team 2018, arXiv e-prints, p. arXiv:1809.09642

Torrey P., Vogelsberger M., Genel S., Sijacki D., Springel V., Hernquist L., 2014, Mon. Not. R. Astron. Soc., 438, 1985

Tremmel M., Governato F., Volonteri M., Pontzen A., Quinn T. R., 2018, Astrophys. J. Lett., 857, L22

Tsai C.-W., et al., 2013, Astrophys. J., 779, 41

Tsalmantza P., Decarli R., Dotti M., Hogg D. W., 2011, Astrophys. J., 738, 20

Urry C., 2004, AGN Unification: An Update. p. 49

Urry C. M., Padovani P., 1995, PASP, 107, 803

Valtonen M. J., et al., 2008, Nature, 452, 851

Vogelsberger M., Genel S., Sijacki D., Torrey P., Springel V., Hernquist L., 2013, Mon. Not. R. Astron. Soc., 436, 3031

Vogelsberger M., et al., 2014a, Mon. Not. R. Astron. Soc., 444, 1518

Vogelsberger M., et al., 2014b, Nature, 509, 177

Volonteri M., Miller J. M., Dotti M., 2009, Astrophys. J. Lett., 703, L86

Wang L., Greene J. E., Ju W., Rafikov R. R., Ruan J. J., Schneider D. P., 2017, Astrophys. J., 834, 129

Wang J.-M., Songsheng Y.-Y., Li Y.-R., Yu Z., 2018, Astrophys. J., 862, 171

XRISM Science Team 2020, arXiv e-prints, p. arXiv:2003.04962

Yu Q., Lu Y., 2001, Astron. Astrophys., 377, 17

van der Walt S., Colbert S. C., Varoquaux G., 2011, CoRR, 1102.1523 
APPENDIX A: ADDITIONAL MATERIAL

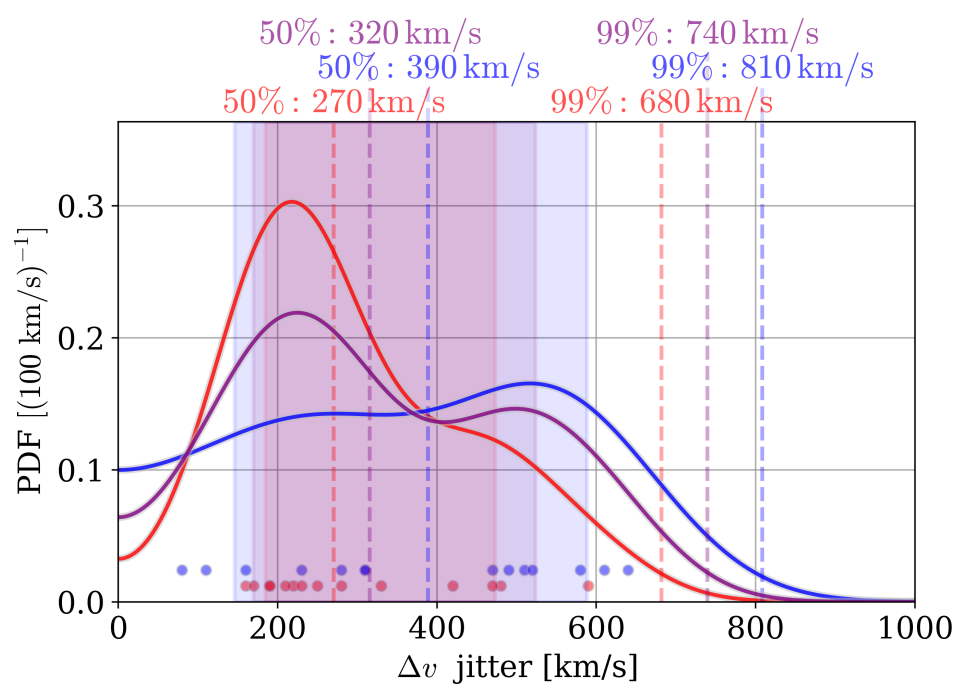

Figure A1: Distribution of velocity-offset variations ('jitters') from (Doan et al. 2019), for the red and blue components of the observed BLs. Measurements are shown as ticks at $y=0$, and KDE distributions are shown using a Scott's factor bandwidth, and Gaussian kernel. The purple curve is the KDE distribution taking both red and blue data as independent. The KDE 50\% (median) and 99\% (Gaussian standard deviation $\sigma \approx 2.3$ ) jitter for each component are shown with dashed vertical lines and corresponding labels.

\begin{tabular}{|c|c|c|c|c|c|c|c|}
\hline & & & $5 \%$ & $25 \%$ & $50 \%$ & $75 \%$ & $95 \%$ \\
\hline \multirow{6}{*}{ All } & \multirow{6}{*}{ - } & $M\left[M_{\odot}\right]$ & $3.4 \times 10^{6}$ & $7.2 \times 10^{6}$ & $1.8 \times 10^{7}$ & $6.0 \times 10^{7}$ & $4.1 \times 10^{8}$ \\
\hline & & $\mathrm{q}$ & $2.9 \times 10^{-2}$ & $1.7 \times 10^{-1}$ & $4.0 \times 10^{-1}$ & $6.8 \times 10^{-1}$ & $9.3 \times 10^{-1}$ \\
\hline & & $\mathrm{z}$ & 0.24 & 0.41 & 0.53 & 0.62 & 0.69 \\
\hline & & $p[\mathrm{yr}]$ & $1.4 \times 10^{2}$ & $1.5 \times 10^{3}$ & $9.6 \times 10^{3}$ & $4.0 \times 10^{4}$ & $2.0 \times 10^{5}$ \\
\hline & & $a[\mathrm{pc}]$ & $2.1 \times 10^{-2}$ & $1.3 \times 10^{-1}$ & $4.9 \times 10^{-1}$ & $1.3 \times 10^{0}$ & $4.1 \times 10^{0}$ \\
\hline & & $a\left[r_{g}\right]$ & $1.2 \times 10^{4}$ & $1.4 \times 10^{5}$ & $4.5 \times 10^{5}$ & $1.2 \times 10^{6}$ & $4.1 \times 10^{6}$ \\
\hline \multirow{6}{*}{$\begin{array}{l}\text { Secondary } \\
\text { Offset } \\
\left(v_{\text {off }}\right)\end{array}$} & \multirow{6}{*}{$0.49 \%$} & $M\left[M_{\odot}\right]$ & $1.4 \times 10^{8}$ & $4.0 \times 10^{8}$ & $7.6 \times 10^{8}$ & $1.4 \times 10^{9}$ & $3.7 \times 10^{9}$ \\
\hline & & q & $9.6 \times 10^{-4}$ & $4.2 \times 10^{-3}$ & $1.2 \times 10^{-2}$ & $3.6 \times 10^{-2}$ & $1.5 \times 10^{-1}$ \\
\hline & & $\mathrm{z}$ & 0.25 & 0.42 & 0.54 & 0.63 & 0.69 \\
\hline & & $p[\mathrm{yr}]$ & $8.3 \times 10^{2}$ & $1.5 \times 10^{3}$ & $2.3 \times 10^{3}$ & $3.7 \times 10^{3}$ & $9.0 \times 10^{3}$ \\
\hline & & $a[\mathrm{pc}]$ & 0.22 & 0.41 & 0.58 & 0.87 & 1.82 \\
\hline & & $a\left[r_{g}\right]$ & $5.0 \times 10^{3}$ & $1.1 \times 10^{4}$ & $1.8 \times 10^{4}$ & $2.9 \times 10^{4}$ & $4.9 \times 10^{4}$ \\
\hline \multirow{6}{*}{$\begin{array}{l}\text { Secondary } \\
\text { Changing } \\
(\Delta v)\end{array}$} & \multirow{6}{*}{$0.027 \%$} & $M\left[M_{\odot}\right]$ & $2.9 \times 10^{8}$ & $8.7 \times 10^{8}$ & $1.6 \times 10^{9}$ & $2.7 \times 10^{9}$ & $5.5 \times 10^{9}$ \\
\hline & & q & $3.9 \times 10^{-4}$ & $7.3 \times 10^{-4}$ & $2.2 \times 10^{-3}$ & $3.8 \times 10^{-3}$ & $9.1 \times 10^{-3}$ \\
\hline & & $\mathrm{z}$ & 0.25 & 0.37 & 0.50 & 0.59 & 0.68 \\
\hline & & $p[\mathrm{yr}]$ & $5.2 \times 10^{2}$ & $7.0 \times 10^{2}$ & $8.6 \times 10^{2}$ & $1.1 \times 10^{3}$ & $1.4 \times 10^{3}$ \\
\hline & & $a[\mathrm{pc}]$ & 0.19 & 0.30 & 0.39 & 0.49 & 0.73 \\
\hline & & $a\left[r_{g}\right]$ & $2.5 \times 10^{3}$ & $3.7 \times 10^{3}$ & $5.6 \times 10^{3}$ & $8.0 \times 10^{3}$ & $1.5 \times 10^{4}$ \\
\hline \multirow{6}{*}{$\begin{array}{l}\text { Secondary } \\
\text { Both } \\
\left(v_{\text {off }} \& \Delta v\right)\end{array}$} & \multirow{6}{*}{$0.014 \%$} & $M\left[M_{\odot}\right]$ & $4.4 \times 10^{8}$ & $1.2 \times 10^{9}$ & $1.8 \times 10^{9}$ & $3.0 \times 10^{9}$ & $6.1 \times 10^{9}$ \\
\hline & & $\mathrm{q}$ & $3.6 \times 10^{-4}$ & $6.2 \times 10^{-4}$ & $1.8 \times 10^{-3}$ & $3.1 \times 10^{-3}$ & $5.7 \times 10^{-3}$ \\
\hline & & $\mathrm{z}$ & 0.25 & 0.37 & 0.48 & 0.58 & 0.68 \\
\hline & & $p[\mathrm{yr}]$ & $5.2 \times 10^{2}$ & $7.0 \times 10^{2}$ & $8.4 \times 10^{2}$ & $1.0 \times 10^{3}$ & $1.4 \times 10^{3}$ \\
\hline & & $a[\mathrm{pc}]$ & 0.22 & 0.33 & 0.41 & 0.51 & 0.76 \\
\hline & & $a\left[r_{g}\right]$ & $2.3 \times 10^{3}$ & $3.3 \times 10^{3}$ & $4.8 \times 10^{3}$ & $6.6 \times 10^{3}$ & $1.1 \times 10^{4}$ \\
\hline
\end{tabular}

Table A1: Parameters of detectable binary systems with redshift $z<0.7$. The indicated quantiles are given for total mass $(M)$, mass ratio $(q)$, separation $(a)$, and orbital period $(p)$. In only four binaries $\left(2 \times 10^{-8}\right.$ of systems $)$ is the primary detectable, and those have parameters: $M \approx 2-5 \times 10^{9} \mathrm{M}_{\odot}, \quad q \approx 0.7-0.9, \quad z \approx 0.6-0.8, \quad a \approx 2.2-2.8 \mathrm{pc}$. 